\title{
Expected Option Returns
}

\section{and the Structure of Jump Risk Premia}

Nicole Branger§
Alexandra Hansis*

Christian Schlag $\ddagger$

This version: September 4, 2008

\begin{abstract}
The paper analyzes expected option returns in a model with stochastic volatility and jumps. A comparison with empirically documented returns shows that the ability of the model to explain these returns can differ significantly depending on the holding period and depending on whether we consider call or put options. Furthermore, we show that the size of the jump risk premium and its decomposition into a premium for jump intensity risk, jump size risk, and jump variance risk has a significant impact on expected option returns. In particular, expected returns on OTM calls can even become negative if e.g. jump variance risk is priced.
\end{abstract}

Keywords: Option returns, put puzzle, jump risk premia, volatility risk premium

JEL: G13

\footnotetext{
§Finance Center Münster, University of Münster, Universitätsstr. 14-16, D-48143 Münster, Germany. E-mail: nicole.branger@wiwi.uni-muenster.de

*Graduate Program 'Finance and Monetary Economics', Goethe University, Mertonstr. 1721/Uni-Pf 77, D-60054 Frankfurt am Main, Germany. E-mail: hansis@finance.uni-frankfurt.de

${ }_{\ddagger}^{\ddagger}$ Finance Department, Goethe University, Mertonstr. 17-21/Uni-Pf 77, D-60054 Frankfurt am Main, Germany. E-mail: schlag@finance.uni-frankfurt.de

Earlier versions of this paper were presented at the Fifth World Congress of the Bachelier Finance Society in London 2008. The authors would like to thank the conference participants for useful comments and suggestions.
} 


\section{Introduction and Motivation}

The returns on put options observed in the market are negative and very large in absolute terms. Holding an at the money (ATM) or out-of-the-money (OTM) put over one month may well give a monthly return of up to $-40 \%$ or $-90 \%$ on average, as e.g. documented by Bondarenko (2003) or Broadie, Chernov, and Johannes (2008). While we expect average returns on puts to be negative, and while we also expect them to be rather large in absolute value, since OTM puts provide an insurance against crashes, the absolute size of these returns is still puzzling. This seems to imply that selling put options is a very good investment (despite the potentially large losses from time to time), and Driessen and Maenhout (2007) indeed find that nearly all investors should have short positions in puts. The returns of these options thus seem rather extreme, or, stated differently, puts seem to be significantly overpriced. That is what is known as the put puzzle.

In response to these potential anomalies, Broadie, Chernov, and Johannes (2008) provide a detailed analysis of average option returns when the options are held until maturity (maturity returns). Their main finding is that put returns are not as puzzling as they seem at a first glance, but can well be explained in standard option pricing models. They use Monte Carlo simulation to determine the finite sample distribution of average put returns, and they find that due to the large standard errors, empirical average put returns would even be in line with the Black-Scholes model. Returns on option portfolios, for which the variability is reduced, reject the models of Black-Scholes and Heston (1993), but are perfectly in line with a stochastic volatility model with jumps (SVJ model), once jump risk premia are included. The authors' overall conclusion is therefore that put (and, more generally, option) returns are not as puzzling as they previously seemed.

In our paper, we provide a detailed analysis of option returns beyond maturity returns by considering also intermediate returns on options which are sold before maturity, e.g. the return which is earned by buying an option with two months to maturity and selling it one month later. With the holding period, we add another dimension to moneyness and time to maturity and argue that these intermediate 
returns provide additional information about the underlying model. From a theoretical point of view, this is because these returns depend on the joint distribution of the stock price and volatility instead of just on the distribution of the stock price. From an empirical point of view, we show, by comparing our simulation results to the option returns documented by Coval and Shumway (2001) and Broadie, Chernov, and Johannes (2008), that models may well be in line with maturity returns, but are still not able to explain intermediate returns.

As emphasized in Broadie, Chernov, and Johannes (2008) risk premia are important in matching the empirically observed patterns in average option returns. The theoretical contribution of our paper is therefore to analyze the impact of the exact structure of the risk premia on expected option returns. In particular, we focus on the jump risk premium, where jump intensity risk, jump size risk, and jump variance risk might be priced, i.e. these quantities may be different under the $\mathbb{P}$ - and the $\mathbb{Q}$-measure. We show that the decomposition of this premium into these sources of risk indeed has a significant impact on expected option returns. This impact goes so far that expected (local) returns on call options can even become negative, depending on whether or not we include a risk premium for jump variance risk. This 'fine structure' of risk premia thus matters a lot when analyzing option returns. Option returns might thus also allow to identify which risk factors are priced.

For the first part of our analysis we use the simulation setup suggested by Broadie, Chernov, and Johannes (2008) to allow for a direct comparison of the results. We consider the maturity and intermediate returns on call and put options. The empirically observed average returns are taken from the studies by Broadie, Chernov, and Johannes (2008) and Coval and Shumway (2001).

When the equity risk premium is composed by a premium for stock diffusion risk and an additional premium for jump risk, we need further assumptions on the relative size of diffusion and jump risk premia, and we have to know whether jump intensity risk, jump size risk, and/or jump variance risk are priced. In a first step, we follow Broadie, Chernov, and Johannes (2008) by using the general equilibrium models developed by Bates (1988) and Naik and Lee (1990). This results in a pre- 
mium for jump intensity risk and for (mean) jump size risk, i.e. both the expected frequency of jumps and the expected size of a jump differ between the $\mathbb{P}$ - and the $\mathbb{Q}$-measure. Under this scenario the model is able to explain maturity put returns very well as shown by Broadie, Chernov, and Johannes (2008), whereas the empirically observed average intermediate put returns cannot be matched, with p-values for the test of equality between theoretical mean and observed average below $1 \%$ in most cases. Furthermore, one cannot simply generalize findings for put returns to calls, although this is true for the prices of these options due to put-call parity. A model may thus well be in line with put returns, but not with call returns, or vice versa.

In the second part of our analysis we focus on the question how the composition of the total jump risk premium affects the properties of expected option returns. We calculate the option returns for several restrictions of the basic models, where we set the volatility risk premium, the premium for jump variance, or both equal to zero. We find that average put returns are rather insensitive with respect to how the jump risk premium is specified. Call returns, however, exhibit a significant exposure to the structure of the jump risk premium. In most cases, average call returns can even become negative for certain ranges of strikes.

To get the intuition for the results, we consider the expected local returns over the next infinitesimal interval of length $\mathrm{dt}$ and decompose them into the returns due to risk premia for stock and volatility diffusion risk, jump intensity risk, jump size risk, jump variance risk, and a risk premium due to jumps in variance. The analysis shows that the main reason for negative expected call returns in our model is a premium for jump variance risk, where jumps have a larger variance under the $\mathbb{Q}$-measure than under the $\mathbb{P}$-measure. A premium for jumps in variance and a jump intensity premium, combined with not too negative jumps, can also lead to negative expected call returns. While these results seem very surprising at first sight, they also highlight the importance of the structure of jump risk premia, which cannot be extracted reliably from a cross-section of prices, but for which one needs the time-series information from returns as well. 
An important implication of our analysis considers the identification of option pricing models with jump components. Jumps are rare events, and parameters of the jump distribution in particular under the physical measure are hard to estimate. This leads to a significant model risk, which is relevant, e.g., when we consider optimal investment strategies in derivatives. Option returns might help to identify the exact nature of jump risk premia.

Our paper is related to the literature dealing with option returns. Coval and Shumway (2001) and Jones (2006) analyze option returns and conclude that these are not in line with standard one-factor models, but that there are further risk factors and that at least one of these risk factors is priced. Ibánez (2007) considers returns on delta-hedged portfolio and finds that a significant part of the average returns can be explained by a negative volatility risk premium. Driessen and Maenhout (2007) show that most investors would optimally hold large short positions in options and conclude that options are overpriced, or, stated differently, that option returns are too low. Bollen and Whaley (2004) argue that high prices of OTM puts can be explained by a buying pressure for these options and empirically study the link between put prices and the demand for these puts. Bondarenko (2003) considers a general class of (equilibrium) pricing models and concludes that option returns are not in line with these models. He focuses in particular on intermediate option returns and argues that the true puzzle is that option returns are inconsistent over time.

The remainder of the paper is organized as follows. In Section 2, we introduce the model and the setup of the simulation study. Section 3 analysis option returns in different models and for different holding periods. The impact of the jump risk premium is studied in Section 4. Section 5 concludes. 


\section{Our Methodology}

\subsection{The Model}

We consider a model with stochastic volatility and jumps both in the stock price and in its volatility. The dynamics of the stock price $S$ (or index level) and the local variance $V$ under the physical measure $\mathbb{P}$ are given by

$$
\begin{aligned}
& d S_{t}=\left(r+a-\bar{\mu}^{\mathbb{P}} \lambda^{\mathbb{P}}\right) S_{t} d t+\sqrt{V_{t}} S_{t} d W_{t}^{S, \mathbb{P}}+\left(e^{\xi}-1\right) S_{t} d N_{t} \\
& d V_{t}=\kappa^{\mathbb{P}}\left(\theta^{\mathbb{P}}-V_{t}\right) d t+\sqrt{V_{t}} \sigma_{V}\left(\rho d W_{t}^{S, \mathbb{P}}+\sqrt{1-\rho^{2}} d W_{t}^{V, \mathbb{P}}\right)+\Psi d N_{t} .
\end{aligned}
$$

$W_{t}^{S, \mathbb{P}}$ and $W_{t}^{V, \mathbb{P}}$ are independent Wiener processes. $N_{t}$ is a Poisson process with constant intensity $\lambda^{\mathbb{P}}$. The jump size $\Psi$ in the variance is exponentially distributed with expectation $\mu_{V}^{\mathbb{P}}$, i.e. $\Psi \sim \exp \left\{\mu_{V}^{\mathbb{P}}\right\}$. Conditional on the realized variance jump, the jump size $\xi$ in the stock return follows a normal distribution: $\xi \sim \mathcal{N}\left(\mu_{S}^{\mathbb{P}}+\rho_{J}^{\mathbb{P}} \Psi,\left(\sigma_{S}^{\mathbb{P}}\right)^{2}\right)$, and the mean jump size in the stock price is

$$
\bar{\mu}^{\mathbb{P}}=\frac{\exp \left\{\mu_{S}^{\mathbb{P}}+\frac{\left(\sigma_{S}^{\mathbb{P}}\right)^{2}}{2}\right\}}{1-\rho_{J}^{\mathbb{P}} \mu_{V}^{\mathbb{P}}}-1 .
$$

We assume that there are no dividend payments, and denote the expected excess return on the stock by $a$. Our SVCJ-model nests several option pricing models. Setting $\lambda=\sigma_{V}=0$ and $V_{t}=\theta$ gives the Black-Scholes model, $\Psi=\sigma_{V}=0$ and $V_{t}=\theta$ gives the Merton (1976) model (SJ). For the Heston (1993) (SV) model, we set $\lambda=0$, and for the SVJ model of Bakshi, Cao, and Chen (1997) and Bates (1996), we set $\Psi=0$.

The dynamics under the risk-neutral measure $\mathbb{Q}$ are

$$
\begin{aligned}
d S_{t} & =\left(r-\bar{\mu}^{\mathbb{Q}} \lambda_{S}^{\mathbb{Q}}\right) S_{t} d t+\sqrt{V_{t}} S_{t} d W_{t}^{S, \mathbb{Q}}+\left(e^{\xi}-1\right) S_{t} d N_{t} \\
d V_{t} & =\kappa^{\mathbb{Q}}\left(\theta^{\mathbb{Q}}-V_{t}\right) d t+\sqrt{V_{t}} \sigma_{V}\left(\rho d W_{t}^{S, \mathbb{Q}}+\sqrt{1-\rho^{2}} d W_{t}^{V, \mathbb{Q}}\right)+\Psi d N_{t} .
\end{aligned}
$$

The mean reversion speed and the mean-reversion level of the variance are given by

$$
\begin{aligned}
\kappa^{\mathbb{Q}} & =\kappa^{\mathbb{P}}+\eta_{V} \\
\kappa^{\mathbb{Q}} \theta^{\mathbb{Q}} & =\kappa^{\mathbb{P}} \theta^{\mathbb{P}}
\end{aligned}
$$


where $\eta_{V}$ denotes the premium for (total) volatility diffusion risk. The intensity of the jump process under $\mathbb{Q}$ is $\lambda^{\mathbb{Q}}$. For the jump sizes, we assume that they still follow an exponential and a normal distribution, respectively, but that all parameters of these distributions may change:

$$
\begin{aligned}
\Psi & \sim \exp \left\{\mu_{V}^{\mathbb{Q}}\right\} \\
\xi & \sim \mathcal{N}\left(\mu_{S}^{\mathbb{Q}}+\rho_{J}^{\mathbb{Q}} \Psi^{\mathbb{Q}},\left(\sigma_{S}^{\mathbb{Q}}\right)^{2}\right)
\end{aligned}
$$

In the following, we will set $\rho_{J}^{\mathbb{Q}} \equiv 0$.

The drift of the stock price depends on the risk-free rate $r$, the compensator for the jump term, and the equity risk premium $a$. The latter is given by

$$
a=\eta_{S} \theta^{\mathbb{P}}+\lambda^{\mathbb{P}} \bar{\mu}^{\mathbb{P}}-\lambda^{\mathbb{Q}} \bar{\mu}^{\mathbb{Q}}
$$

and can be decomposed into a premium for stock diffusion risk and stock jump risk. The parameters under the physical measure are summarized as $\Theta^{\mathbb{P}}$, the parameters under the risk-neutral measure are summarized in $\Theta^{\mathbb{Q}}$.

For the following simulations, we will use two different parameterizations, which are based on the time series of stock prices only (first case) and on the time series of stock prices as well as on the cross section of option prices (second case). This also allows us to see whether the use of option prices in the calibration helps to explain option returns better. In the first case we rely on the estimates of Broadie, Chernov, and Johannes (2008) (hereafter BCJ08) which are solely based on historical index return data. The risk premia for stock price diffusion and jump risk are derived from the equity risk premium, the remaining risk premia for volatility risk are set equal to zero. For their parameter calibration BCJ08 use daily S\&P 500 index returns from 1987 to 2005. For the sake of clarity, we will refer to this parameterization as time-series based parameterization. In the second case we use the estimates of Eraker, Johannes, and Polson (2003) (hereafter EJP) for the physical measure and the corresponding estimates of Broadie, Chernov, and Johannes (2007) (hereafter BCJ07) for the risk-neutral measure. EJP estimate the models using S\&P 500 index returns from January 2, 1980, to December 31, 1999. The $\mathbb{Q}$-measure estimates in 
BCJ07 are based on the cross section of option prices on the S\&P 500 futures from January 1987 to March 2003. We will therefore call this parameterization option based parameterization.

The parameters for both parameterizations can be found in Table 1, where we have converted all parameters to annual values. Note that EJP set the drift of $S$ under the physical measure equal to $b+0.5 V_{t}$, i.e.

$$
b+0.5 \theta^{\mathbb{P}}+\lambda^{\mathbb{P}} \bar{\mu}^{\mathbb{P}}=r+\eta_{S} \theta^{\mathbb{P}}+\lambda^{\mathbb{P}} \bar{\mu}^{\mathbb{P}}-\lambda^{\mathbb{Q}} \bar{\mu}^{\mathbb{Q}}
$$

where we rely on the average volatility $\theta^{\mathbb{P}}$ for equating the two drift specifications.

\subsection{Methodology of the Simulation Study}

We use the same simulation setup as Broadie, Chernov, and Johannes (2008) to allow for a direct comparison of the results. We simulate average option returns over $\mathrm{N}=215$ months. The finite sample distributions of these average returns are based on $\mathrm{G}=25,000$ runs, and we take the averages of the average returns as estimate for the mean option returns.

We consider the returns of calls and puts. The times to maturity of the options are one months and two months, and the holding period is set to one month. If the options are held till maturity, we obtain maturity returns, while a holding period below the time to maturity gives intermediate returns.

The empirical returns are taken from the studies of Broadie, Chernov, and Johannes (2008) and Coval and Shumway (2001). Broadie, Chernov, and Johannes (2008) consider the maturity returns on options on S\&P500 futures options with a time to maturity of one month over the period from August 1987 to June 2005. Additionally, they consider the returns on option portfolios like straddles, delta-hedged puts, and crash-neutral straddles and puts. The differences between index options and futures options are rather small, as e.g. argued in Bollen and Whaley (2004) and Bondarenko (2003), and given the absolute size of the returns, will not change the results significantly. Coval and Shumway (2001) consider weekly intermediate 
returns of options on the S\&P500 over a six-year period from January 1990 to October 1995. The remaining time to maturity of the options is between 20 and 50 days, and Coval and Shumway (2001) take the average over these option returns. We convert the weekly returns from their study into monthly returns. ${ }^{1}$

To assess whether an observed empirical return could have been generated by a specific model, we calculate p-values. The p-value is the probability that a return generated by a given model is more extreme than the respective one observed in the market, given that the null hypothesis is true, namely that the market return has indeed been generated by the model under consideration. The p-values below are always given in $\%$.

\section{Maturity Returns versus Intermediate Returns}

The expected return on an option depends on the initial price of the option and the distribution of the payoff (if the option is held till maturity) or the price (if the option is sold before maturity) at the end of the holding period. To explain maturity returns for options with different moneyness levels and different times to maturity, it is thus not enough that the option pricing model matches the initial prices of the options. It must also fit the distributions of the future stock prices at the maturity dates, which determine the distributions of the future payoffs from the options.

Intermediate returns add another dimension to the problem. The price at the end of the holding period depends on the stock price and on further state variables, in our case stochastic volatility. The model must thus fit the joint distributions of the future stock prices and local volatilities at the end of the holding periods. Furthermore, the pricing formula that fits option prices today must also be able to explain future option prices. These additional requirements imply that intermediate returns will provide further information in addition to what we know from maturity returns.

\footnotetext{
${ }^{1}$ Note that we give the average returns as a function of moneyness, while Coval and Shumway (2001) give them depending on the absolute difference between the strike and the index level.
} 
In the following, we consider option returns over a holding period of one month. The options have a time to maturity of one month (resulting in maturity returns) and two months (resulting in intermediate returns). For the simulations, we use the time-series based parametrization. The $\mathbb{P}$-parameters for the simulations are based on the estimates by Broadie, Chernov, and Johannes (2008) for the Black-Scholes, Merton, Heston, and SVJ model.

\subsection{Case 1: Only Stock Diffusion Risk is Priced}

To calculate option prices and option returns, we also need to know the parameters under the risk-neutral measure $\mathbb{Q}$. We follow Broadie, Chernov, and Johannes (2008) and assume in a first step that only stock diffusion risk is priced. The market price $\eta_{S}$ of stock diffusion risk then follows from the equity risk premium in Equation (1), all other market prices of risk are set equal to zero. Figure 1 shows the average option returns for call and put options. The p-values are given in Tables 3 to 5 .

The models yield quite similar returns for ITM options, but differ significantly for OTM options. That is true for OTM puts, where also the option prices calculated in the models will be very different, but it holds also for OTM calls, where the option prices differ much less across the models. Furthermore, the differences between the models are the more pronounced the shorter the time to maturity of the options is, which can partly be attributed to the smaller initial prices.

The model of Black-Scholes gives the smallest returns for OTM call options and the most negative returns for OTM put options. To explain this finding, note that the other option pricing models all generate a smile. They thus give larger prices and larger expected future payoffs (or prices in case of intermediate returns) for OTM puts. However, the absolute difference between the price today and the expected payoff or price in the future turns out to remain nearly constant. The expected return in model $i$ is

$$
\text { Exp. return }{ }^{i}=\frac{\text { Exp. } \text { price }_{1}^{i}-\text { Price }_{0}^{i}}{\text { Price }_{0}^{i}}
$$

and given that the numerator is nearly constant, the return is decreasing in absolute 
terms in the model price today. The negative return of puts is thus largest in absolute terms for the Black-Scholes model, which gives the lowest price for OTM puts. A similar argument holds for OTM calls, and the expected return is smallest for the model of Black-Scholes, which now gives the largest price for OTM calls.

The results for the SJ model differ for calls and puts. For calls, the returns are rather similar to those in the Black-Scholes model, which is no longer true for puts. Adding downward jumps increases the probability that the puts will end in the money, which in turn increases their future payoffs. Since the price increases less (remember that jump risk is not priced in the current parametrization), the returns increase. For calls, on the other hand, jumps that are on average negative do not have much influence on the probability of reaching a very high stock price. As a result the call returns generated by the SJ model are more or less the same as within the Black-Scholes model.

The results for expected returns generated by the SVJ model also differ for calls and puts. While the returns for calls are rather similar to those generated by the SV model, the put returns are close to those in the SJ model. Downward jumps are thus more important for the returns on OTM puts, while the returns on call options are mainly influenced by stochastic volatility. To explain the impact of stochastic volatility on option returns, note that the correlation between the stock price and its volatility is negative. There is thus more probability mass for low future stock prices and less for higher stock prices. Thus, the prices of OTM calls decrease, yielding a higher expected return as compared to Black-Scholes, while the prices of OTM puts increase, which leads to put returns that are lower in absolute terms.

In the next step, we compare the simulated returns to the empirical maturity put returns from Broadie, Chernov, and Johannes (2008), and to the intermediate put and call returns taken from Coval and Shumway (2001). These empirical returns are also plotted in Figure 1. The observed maturity put returns are much more negative than all returns generated by the models. As pointed out by Broadie, Chernov, and Johannes (2008), however, the high variability of these put returns leads to p-values that go up to $13 \%$ (see Table 5), and none of the models can be rejected based on put 
returns. Observed intermediate call returns are much closer to those generated by the models. The p-values given in Table 3 are between $20 \%$ and $45 \%$, so that all models are perfectly in line with the empirical findings. The picture changes, however, if we consider intermediate put returns. Empirical returns are much more negative than those generated by the models. Even the high volatility of the generated returns cannot help to explain the findings, and the p-values in Table 4 are around zero.

Even though the models are able to explain monthly maturity returns on puts, they are still not able to explain monthly intermediate returns on puts. This confirms that maturity returns and intermediate returns indeed contain different information about the price processes under the true measure and about the risk premia. Furthermore, the results show that a model may well be in line with call returns, but still not be able to explain put returns for the same time to maturity and the same holding period. To get the intuition, consider the relation between call and put returns

$$
P_{0}\left(r_{P}-r_{B}\right)+S_{0}\left(r_{S}-r_{B}\right)=C_{0}\left(r_{C}-r_{B}\right)
$$

where $r_{C}, r_{P}, r_{S}$, and $r_{B}$ are the returns on the call, put, stock, and a bond respectively. To convert the call return into a put return and vice versa, we do not only need to know the model-independent returns on the stock and the bond, but also the model dependent prices of calls and puts. Even if a model is (by chance) in line with call returns, it may give incorrect prices for calls and/or puts. This will lead to put returns which are not in line with empirical findings any more.

\subsection{Case 2: Jump Risk is Priced}

In the literature there is ample evidence that other risk factors besides stock (diffusion) risk are priced. Coval and Shumway (2001) state that evidence points towards additional priced risk factors. Bakshi and Kapadia (2003) find that the volatility risk premium contributes significantly to higher prices for calls and puts. Buraschi and Jackwerth (2001) concur, suggesting that more than one priced risk factor is necessary to explain option prices. Benzoni (2002), Chernov and Ghysels (2000), Jones 
(2003), and Pan (2002) also find large volatility risk premia. Additionally there is growing evidence on the presence of jumps in stock returns and volatility (see e.g. Eraker, Johannes, and Polson (2003)), while tests using option data disagree on the importance of jumps in prices and in particular in volatility. ${ }^{2}$ Jones (2006) finds that volatility and jump risk premia alone are not sufficient to explain observed average returns.

In this section, we focus on jump risk premia, while we set the volatility risk premium equal to zero. The equity risk premium is thus explained by a premium for stock diffusion risk and an additional premium for jump risk. We then need an assumption on the relative size of diffusion and jump risk premia, and we need to decide on whether jump intensity risk, jump size risk, or jump variance risk are priced. We follow the time-series based parameterization of Broadie, Chernov, and Johannes (2008), who use the functional forms of the market prices of jump risk as specified in the general equilibrium models of Bates (1988) and Naik and Lee (1990). With the assumption that the relative risk aversion $\gamma$ is 10 , we get a premium for jump intensity risk and for jump size risk, while jump variance risk is not priced. The parameters under the $\mathbb{Q}$-measure are given in Table 2 .

Risk premia are identical in the SJ and in the SVJ model. The resulting option returns are also rather similar, as can be seen in Figure 2. The returns on calls are slightly lower than in the case where only stock diffusion risk is priced, with the exception of maturity returns on deep OTM calls. Returns on puts, which provide an insurance against large downward movements in the stock price, change much more. With a premium for downward jumps, in particular the returns on OTM puts are significantly more negative. The average monthly return on an OTM put with a moneyness of $85 \%$ drops to around $-40 \%$ for intermediate returns and around $-80 \%$ for maturity returns.

To get the intuition for the impact of the jump risk premium, Figure 10 gives the densities for the stock price after one month under the physical measure, the

\footnotetext{
${ }^{2}$ Bakshi, Cao, and Chen (1997) find substantial benefits from including jumps in prices, whereas Bates (2000) and others find that such benefits are economically small, if not negligible.
} 
risk-neutral measure when only stock diffusion risk is priced, and the risk-neutral measure when both stock diffusion risk and stock jump risk are priced. The jump risk premium increases the risk-neutral probability for low stock prices significantly. Therefore, the prices of OTM puts increase significantly, which decreases the already negative returns of put options. Altogether, the returns on OTM puts are way more extreme if there is a jump risk premium, which increases the 'insurance premium' against large downward moves in the stock price.

Since the intermediate returns on calls are lower as in the case where only stock diffusion risk is priced, the difference to the mean returns documented by Coval and Shumway (2001) is larger. The p-values as shown in Table 3, however, are still between $2 \%$ and $39 \%$, and the models are therefore in line with the empirical findings. Furthermore they are now also able to explain the very negative empirical maturity put returns (the p-values are given in Table 5). This evidence points toward the inclusion of jump risk premia into the models. Intermediate put returns, however, are still not in line with the models, even if jump risk is priced. Table 4 shows that the p-values are still below $1 \%$.

\subsection{Summary}

As pointed out by Broadie, Chernov, and Johannes (2008), put returns observed in the market are rather volatile. This leads to large standard errors, making it very difficult to reject an option pricing model based on its inability to explain the very large negative option returns. Nevertheless, there is still clear evidence that jump risk should be priced.

Our results additionally show that tests based on option returns should not only consider options with different moneyness levels and different times to maturity, but also different holding periods of the options. While the models considered are (at least partially) in line with maturity put returns, intermediate put returns are still a challenge and cannot be explained.

Furthermore, our results show that there is a difference between call and put 
returns. It may well be the case that a model is in line with empirical call returns, but not with put returns, or vice versa. Again, this situation can not happen if we consider option prices, since a perfect fit to call prices implies by put-call-parity a perfect fit to put prices, too, given the (trivial) condition that the stock and the bond are priced correctly.

Equation (3), however, shows that a similar parity relation does not hold for returns. Given that a model perfectly explains returns on calls (and, trivially, is in line with stock and bond returns), it explains put returns only if it also explains put prices. Stated differently, we can force a model to perfectly match call returns by 'adjusting' the prices of calls in the appropriate way. This manipulation, however, will not help to explain the returns on puts.

\section{Jump Risk Premia}

We have seen in the last section that the decomposition of the equity risk premium into a diffusion risk premium and a jump risk premium has a significant impact on expected option returns. Now, we take a closer look at the structure of the jump risk premium, which can be further decomposed into a premium for jump size risk, jump variance risk, and jump intensity risk. It will turn out that the impact of this decomposition onto expected option returns, in particular call returns, can be rather dramatic. Additionally, we analyze the impact of the variance risk premium, both for diffusion risk and jump risk, which was set equal to zero in the preceding section.

The analysis relies on the option based parameterization. Starting from the $\mathbb{P}$ parameters as estimated by Eraker, Johannes, and Polson (2003) and the risk-neutral measure as estimated by Broadie, Chernov, and Johannes (2007), we consider several restricted versions of the SVCJ-model and the nested models. In the restricted versions, some risk premia are set equal to zero. 


\subsection{Option Returns}

We now additionally allow for a volatility risk premium. Furthermore, the decomposition of the equity risk premium into a diffusion risk premium and a jump risk premium and the decomposition of the jump risk premium into its components is determined by the cross section of option prices. The premium for jump intensity risk is assumed to be zero.

Figure 3 gives the expected returns for the unrestricted versions of the models. Since the total equity risk premium is larger than in the time-series based calibration used in the preceding section, the option returns in the Black-Scholes model are more extreme as compared to the results in Section 3. The volatility risk premium estimated by Broadie, Chernov, and Johannes (2007) in the Heston model is positive. Since both call and put options have a positive exposure to volatility risk, the resulting returns are larger than in the case without a volatility risk premium. The differences to the Black-Scholes returns are therefore larger, too. The positive volatility risk premium even causes returns on deep OTM puts to be rather close to zero, which is the opposite of what we observe in the market. A positive volatility risk premium thus makes it even more difficult to explain empirical option returns.

The volatility risk premium is positive in the SVJ and SVCJ model, too. Additionally, we include a premium for jump size risk and - different from the analysis in Section 3.2 - a premium for jump volatility risk. Figure 3 shows that the put returns generated in these models are much more negative than the returns generated by the Black-Scholes and the Heston model. In particular, deep OTM puts now have clearly the most negative returns, which are around twice as large a the ones in the model of Black-Scholes. This again shows the importance of including jump risk premia into the models. The behavior of call returns, however, is puzzling. Both maturity and intermediate call returns for deep OTM calls are negative on average. We will come back to this finding in the next section.

The comparison with empirically observed returns, which are given in Figure 3, shows a similar pattern as before. As can also be seen from the p-values in Table 5, the models are agin in line with the maturity put returns. For the Black-Scholes and 
the Heston model, for which simulated returns are rather different from observed returns, this is mainly due to the large standard error and thus the low information content of maturity put returns, as also pointed out by Broadie, Chernov, and Johannes (2008). Intermediate call returns are explained reasonably well by all models (see Table 3), even if the negative expected returns in the SVJ and SVCJ model raise some fundamental questions. Intermediate put returns, however, are still not in line with the models. The p-values in Table 4 are rarely above $1 \%$.

\subsection{A Further Look at Jump Risk Premia}

We now turn to the puzzling result from Section 4.1, namely the negative expected returns on call options for those models that include jumps in the stock price. The main difference to the cases analyzed in Section 3 is how jump risk premia are specified. While in Section 3, jump intensity risk and jump size risk are priced, we now consider premia for jump size risk and jump variance risk. The large changes in the size and in particular in the sign of option returns show that the exact decomposition of jump risk premia has a significant effect on option returns.

For a further analysis of the impact the fine structure of risk premia has on expected option returns, we consider several restricted versions of our models, where we set the diffusive volatility risk premium, the premium for jump variance risk, or both equal to zero. The resulting returns in the SVJ and SVCJ model are shown in Figures 4 and 7, respectively. Put returns are rather insensitive against how the jump risk premium is specified. Returns on OTM calls, however, change significantly depending on the restrictions. The only model where call returns do not eventually become negative is the SVJ model in which the premium for jump variance risk is set to zero.

To get the intuition for this rather puzzling behavior of call returns, we analyze expected local returns. They depend on the exposures of the options to stock price diffusion risk, volatility diffusion risk, and jump risk, as well as on the market prices of risk for these factors. Using Itô and the fundamental partial differential equation, 
the expected excess return of an option in the most general SVCJ model is

$$
\begin{aligned}
E^{\mathbb{P}}\left[\frac{d C}{C}-r d t\right]= & \underbrace{\frac{\partial C}{\partial S} \cdot \frac{S}{C} \eta_{S} V}_{E R^{S}} d t+\underbrace{\frac{\partial C}{\partial V} \cdot \frac{1}{C} \eta_{V} V}_{E R^{V}} d t \\
& +\underbrace{\left(\frac{E^{\mathbb{P}}\left[C\left(S e^{X}, V+\psi\right)\right]-C}{C} \lambda^{\mathbb{P}}-\frac{E^{\mathbb{Q}}\left[C\left(S e^{X}, V+\psi\right)\right]-C}{C} \lambda^{\mathbb{Q}}\right)}_{E R^{J}} d t .
\end{aligned}
$$

$E R^{S}$ captures the impact of stock diffusion risk. Since the delta of a call is positive, the sign of this term is equal to the sign of the market price for stock diffusion risk $\eta_{S}$. For some of our calibrations, this market price of risk is negative and is thus the first potential reason for negative call returns. The impact of diffusive volatility risk is captured by $E R^{V}$, and with vega being positive, the sign of this term is equal to the sign of $\eta_{V}$. Finally, $E R^{J}$ captures the impact of jump risk on the expected option returns. It can be further decomposed into ${ }^{3}$

$$
\begin{aligned}
E R^{J}= & \underbrace{\frac{E^{\mathbb{P}}\left[C\left(S e^{X}\right)\right]-C}{C}\left(\lambda^{\mathbb{P}}-\lambda^{\mathbb{Q}}\right)}_{E R_{\lambda}^{J}}+\underbrace{\frac{E^{\mathbb{P}}\left[C\left(S e^{X}\right)\right]-E^{\mathbb{Q}\left(\sigma_{S}^{\mathbb{P}}\right)}\left[C\left(S e^{X}\right)\right]}{C} \lambda^{\mathbb{Q}}}_{E R_{\mu}^{J}} \\
& +\underbrace{\frac{E^{\mathbb{Q}\left(\sigma_{S}^{\mathbb{P}}\right)}\left[C\left(S e^{X}\right)\right]-E^{\mathbb{Q}}\left[C\left(S e^{X}\right)\right]}{C} \lambda^{\mathbb{Q}}}_{E R_{\sigma}^{J}} \\
& +\underbrace{\frac{E^{\mathbb{P}}\left[C\left(S e^{X}, V+\psi\right)\right]-E^{\mathbb{P}}\left[C\left(S e^{X}, V\right)\right]}{C} \lambda^{\mathbb{P}}-\frac{E^{\mathbb{Q}}\left[C\left(S e^{X}, V+\psi\right)\right]-E^{\mathbb{Q}}\left[C\left(S e^{X}, V\right)\right]}{C} \lambda^{\mathbb{Q}}}_{C R_{V}^{J}}
\end{aligned}
$$

where $\mathbb{Q}\left(\sigma_{S}^{\mathbb{P}}\right)$ denotes the jump size distribution with mean $\bar{\mu}^{\mathbb{Q}}$, but old variance $\sigma_{S}^{\mathbb{P}}$. $E R_{\lambda}^{J}$ captures the change in the jump intensity, $E R_{\mu}^{J}$ captures the change in the mean jump size, $E R_{\sigma}^{J}$ captures the change in the volatility of the stock jump, and $E R_{V}^{J}$ captures the jump in volatility.

When analyzing these terms, we make the basic assumption that jumps happen more often and are more severe under the risk-neutral measure than under the true measure, i.e. $\lambda^{\mathbb{Q}} \geq \lambda^{\mathbb{P}}$ and $E^{\mathbb{Q}}\left[e^{X}\right] \leq E^{\mathbb{P}}\left[e^{X}\right] \leq 0$. This results in a positive jump risk premium for the stock, and it is in line with the parameterizations used in our analysis.

\footnotetext{
${ }^{3}$ Note that this decomposition is not unique, but depends on the order in which we go from the physical measure to the risk-neutral measure.
} 
The premium $E R_{\mu}^{J}$ for the mean jump size can be shown to be positive for any convex claim like a call or put. The premium $E R_{\lambda}^{J}$ due to jump intensity risk is more involved. It is positive if the mean size of the downward jumps in the stock, i.e. $E^{\mathbb{P}}\left[e^{X}\right]$, is 'sufficiently' negative, but is positive otherwise. $E R_{\sigma}^{J}$ is the risk premium due to jump variance risk. For $\sigma_{S}^{\mathbb{Q}}>\sigma_{S}^{\mathbb{P}}$, i.e. if jumps are more variable under the risk-neutral measure than under the true measure, this term is negative. For more details and proofs, see Appendix A.1.

Summing up the findings until now, we see that call returns can well be negative. Potential reasons for negative call returns are a negative market price of risk for stock diffusion risk or for volatility diffusion risk, too small downward jumps in the stock price, a premium for variance jump risk where jumps are more variable under the risk-neutral measure, and, finally, volatility jumps.

Table 6, Panel A, gives the signs of local expected call returns for the SV, the SVJ, and the SVCJ model in the unrestricted case as well as when several risk premia are set equal to zero. Furthermore, the table states the signs of the different components of the expected returns due to stock price diffusion risk $\left(E R^{S}\right)$, volatility diffusion risk $\left(E R^{V}\right)$, and jump risk $\left(E R^{J}\right)$. Details on the calculation can be found in Appendix A.2 and A.3. First, note that the market price for stock diffusion risk is negative in some cases. This market price of stock diffusion risk is not determined from the cross section of options prices, but it follows from the equity risk premium and from the contribution of jump risk to the equity risk premium. In particular, it is negative in those models where the premium for jump variance risk is restricted to be zero. The contribution of volatility diffusion risk to the expected call return is non-negative in all cases, since the market price of volatility risk is either estimated to be positive or is assumed to be zero.

Figures 5 and 8 show the contributions of diffusion risk and jump risk to the local expected returns in the SVJ and SVCJ model, respectively. In the SVJ model without any restrictions, stock diffusion risk has a positive contribution to the overall local expected call return. The contribution of volatility diffusion risk, on the other hand, can be neglected, which is true in all restricted versions of the model we 
consider. The contribution of jump risk is negative for moderate to high strike prices and is the largest component in absolute terms, leading to a large negative expected return for high strike prices. If jump variance risk is not priced, the picture changes completely. Now, stock diffusion risk has a negative contribution, while jump risk has a positive one. For the SVCJ, the results are qualitatively similar in the unrestricted case. If the jump variance risk premium is restricted to be equal to zero, however, the jump risk premium does not change sign, but is still negative for most strike prices. Furthermore, restricting the diffusive volatility risk premium to zero now has a larger impact, too, and the remaining components of the return become less extreme in this case.

The contribution of jump risk is more involved. It is positive over all moneyness levels only in the SVJ model when there is no premium for variance jump risk. In all other models, it can become negative. The results for the further decomposition of this premium are given in Table 6, Panel B. Figures 6 and 9 show the contributions of the different jump risk components to the local expected returns in the SVJ and SVCJ model, respectively. The premium for jump intensity risk is zero by assumption, the premium for jump size risk is (since the jumps are negative 'enough') positive in both models and for all restrictions. The premium due to jump variance risk is negative, and, as can be seen in Table 6, Panel A, large enough to induce a negative risk premium on deep OTM calls. The figure shows that this variance risk premium is the driving factor for the total jump risk premium in the SVJ model. In the SVCJ model, on the other hand, the importance of the jump variance risk premium (for jumps in the stock price) is significantly lower. Its role is taken over by the premium for jumps in the volatility of the stock price. In our parameterizations, the mean jump size in the volatility is much larger under $\mathbb{Q}$ than under $\mathbb{P}$, which results in a negative contribution of this component. As the figure shows, this term is the largest one in absolute terms, so that it again leads to a negative expected excess return.

Figure 11 compares the densities of the SVJ model under the physical measure and under the risk-neutral measure in the unrestricted case and in the cases where 
the risk premium on jump variance risk and/or the diffusive volatility risk premium are set equal to zero. If $\sigma_{S}^{\mathbb{Q}}$ is restricted to be equal to $\sigma_{S}^{\mathbb{P}}, \mu_{S}^{\mathbb{Q}}$ becomes more negative, i.e. the premium for jump size risk increases, and at the same time the premium $\eta_{S}$ for stock diffusion risk is negative (see Table 6, Panel B). If we allow for a premium

for jump variance risk, $\sigma_{S}^{\mathbb{Q}}$ is twice as large as $\sigma_{S}^{\mathbb{P}}$. The risk-neutral densities then have much fatter tails than in the restricted case. This effect has the biggest influence on OTM calls and it therefore explains the substantial differences in OTM call returns between the different versions of the model as seen in Figure 4.

The results in Section 3 have shown that we need to include a jump risk premium in order to get closer to observed option returns. The results in this section emphasize how important the exact specification of the jump risk premium is. The generated expected returns are very different, depending on whether jump size risk, jump intensity risk, or jump variance risk are priced.

Jumps are rare events, and therefore parameters of the jump distribution are hard to estimate. This is especially true under the physical measure. Together with the large impact that the exact decomposition of the jump risk premium has on option returns, this leads to significant model risk when considering option returns and also when calculating the optimal investment strategy of an investor who also has access to derivatives. On the other hand, the pronounced dependence of options returns on the fine structure of risk premia implies that option returns might help to identify the exact nature of jump risk premia. The differences across the restricted versions are indeed statistically significant. For details, see Appendix B.1.

\section{Conclusion}

We analyze returns on options in state-of-the-art option pricing models that include stochastic volatility, jumps in the stock price, and jumps in the variance. First, we find that expected option returns differ significantly depending on whether we consider maturity returns or intermediate returns. In particular, while the expected maturity returns generated by the models are in line with empirical observations, 
intermediate returns may not be so. Furthermore, there are significant differences between the ability of the models to explain call and put returns. Models that aim at explaining expected option returns thus do not only have to consider the expected returns for different times to maturity and different levels of moneyness, but also the returns on calls and puts as well as maturity and intermediate returns.

Second, we show that the fine structure of risk premia has a significant impact on option returns. To explain the large negative average returns on puts, a jump risk premium has to be included, as also pointed out by Broadie, Chernov, and Johannes (2008). The exact structure of the jump risk premium is then crucial. We show that this structure has a larger impact on option returns than the choice of the exact model. Depending on whether jump intensity risk, jump size risk, or jump variance risk are priced, call returns differ significantly and can even change sign from positive to negative. Option returns are thus subject to the risk of model mis-specification. On the other hand, option returns might help in identifying risk premia for jumps, which are rare events and therefore particularly hard to estimate.

Given that jump risk premia have a significant impact on expected option returns, the question is whether that is also true for portfolio planning. In further research, we thus want to study the optimal portfolio decision of an investor who has access to derivatives. In particular, we are interested in the impact the decomposition of the jump risk premium has in such a situation. 


\section{A Expected Local Returns}

\section{A.1 Analysis of Components}

In Section 4.2, we decompose the expected option returns and look more closely at the impact of jump risk premia. Details on the calculation of the components and on their signs are given here.

\section{A.1.1 Risk premium for mean jump size risk}

$E R_{\mu}^{J}$ is the risk premium due to the change in the mean jump size. It is given by

$$
\begin{aligned}
E R_{\mu}^{J} & =\frac{E^{\mathbb{P}}\left[C\left(S e^{X}, \ldots\right)\right]-E^{\mathbb{Q}\left(\sigma_{S}^{\mathbb{P}}\right)}\left[C\left(S e^{X}, \ldots\right)\right]}{C} \lambda^{\mathbb{Q}} \\
& =\frac{1}{C} \int_{-\infty}^{\infty}\left[C\left(S e^{\bar{\mu}^{\mathbb{P}}-0.5\left(\sigma_{S}^{\mathbb{P}}\right)^{2}+\sigma_{S}^{\mathbb{P}} x}\right)-C\left(S e^{\bar{\mu}^{Q}-0.5\left(\sigma_{S}^{\mathbb{P}}\right)^{2}+\sigma_{S}^{\mathbb{P}} x}\right)\right] n(x) d x \lambda^{\mathbb{Q}},
\end{aligned}
$$

where $n$ is the density function of the standard normal distribution. For $E^{\mathbb{Q}}\left[e^{X}\right]<$ $E^{\mathbb{P}}\left[e^{X}\right]$ it holds that $E R_{\mu}^{J}>0$.

Proof: For $\bar{\mu}^{\mathbb{P}}>\bar{\mu}^{\mathbb{Q}}$, it holds that

$$
S e^{\bar{\mu}^{\mathbb{P}}-0.5\left(\sigma_{S}^{\mathbb{P}}\right)^{2}+\sigma_{S}^{\mathbb{P}} x}>S e^{\bar{\mu}^{\mathbb{Q}}-0.5\left(\sigma_{S}^{\mathbb{P}}\right)^{2}+\sigma_{S}^{\mathbb{P}} x} .
$$

Since the call price is an increasing function of the stock price, this gives

$$
C\left(S e^{\bar{\mu}^{\mathbb{P}}-0.5\left(\sigma_{S}^{\mathbb{P}}\right)^{2}+\sigma_{S}^{\mathbb{P}} x}\right)>C\left(S e^{\bar{\mu}^{\mathbb{Q}}-0.5\left(\sigma_{S}^{\mathbb{P}}\right)^{2}+\sigma_{S}^{\mathbb{P}} x}\right)
$$

from which we immediately get

$$
E^{\mathbb{P}}\left[C\left(S e^{X}, \ldots\right)\right]-E^{\mathbb{Q}\left(\sigma_{S}^{\mathbb{P}}\right)}\left[C\left(S e^{X}, \ldots\right)\right]>0
$$

\section{A.1.2 Risk premium for jump intensity risk}

$E R_{\lambda}^{J}$ is the risk premium due to the change in the mean jump size. It is given by

$$
E R_{\lambda}^{J}=\frac{E^{\mathbb{P}}\left[C\left(S e^{X}, \ldots\right)-C(S)\right]}{C}\left(\lambda^{\mathbb{P}}-\lambda^{\mathbb{Q}}\right) .
$$

If $\lambda^{\mathbb{Q}}>\lambda^{\mathbb{P}}$ and $E^{\mathbb{P}}\left[e^{X}\right]$ is sufficiently negative, it holds that $E R_{\lambda}^{J}>0$. 
Proof: Consider the special case $E^{\mathbb{P}}\left[e^{X}\right]=0$. The call price is a convex function of the stock price, so that

$$
E^{\mathbb{P}}\left[C\left(S e^{X}, \ldots\right)\right] \geq C\left(E^{\mathbb{P}}\left[S e^{X}\right], \ldots\right)=C(S, . .)
$$

This implies

$$
E^{\mathbb{P}}\left[C\left(S e^{X}, \ldots\right)-C(S)\right] \geq 0
$$

so that $E R_{\mu}^{J} \leq 0$.

In general (and also in our parameterization) jumps are on average negative. $E^{\mathbb{P}}\left[C\left(S e^{X}, \ldots\right)\right]$

is increasing in the mean jump size. When $E^{\mathbb{P}}\left[e^{X}\right]$ is 'sufficiently' negative it therefore follows that

$$
E^{\mathbb{P}}\left[C\left(S e^{X}, \ldots\right)-C(S)\right] \leq 0
$$

\section{A.1.3 Risk premium for jump volatility risk}

$E R_{\sigma}^{J}$ is the risk premium due to the change in the volatility of the stock jump.

$$
E R_{\sigma}^{J}=\frac{E^{\mathbb{Q}\left(\sigma_{S}^{\mathbb{P}}\right)}\left[C\left(S e^{X}, \ldots\right)\right]-E^{\mathbb{Q}}\left[C\left(S e^{X}, \ldots\right)\right]}{C} \lambda^{\mathbb{Q}}
$$

For $\sigma_{S}^{\mathbb{Q}}>\sigma_{S}^{\mathbb{P}}$, it holds that $E R_{\sigma}^{J}<0$.

Proof: Since the call price is convex in the stock price and thus also in $X$, the result follows immediately from the next proposition.

Proposition 1 For a normally distributed random variable $X$, it holds that the expectation $E[g(X)]$ of some function $g$ of $X$ is increasing (decreasing) in the volatility of $X$ if the function $g$ is convex (concave).

Proof: We denote the expectation by $f(\mu, \sigma)$ where $f$ is given by

$$
f(\mu, \sigma)=E[g(X)]=E[g(\mu+\sigma Y)] .
$$

$Y$ follows a standard normal distribution and does therefore neither depend on $\mu$ nor on $\sigma$. The partial derivatives of $f$ are given by

$$
\begin{aligned}
\frac{\partial f}{\partial \sigma}(\mu, \sigma) & =E\left[g^{\prime}(\mu+\sigma Y) Y\right] \\
\frac{\partial^{2} f}{\partial \sigma^{2}}(\mu, \sigma) & =E\left[g^{\prime \prime}(\mu+\sigma Y) Y^{2}\right]
\end{aligned}
$$


For $\sigma=0$, we have

$$
\left.\frac{\partial f}{\partial \sigma}(\mu, \sigma)\right|_{\sigma=0}=E\left[g^{\prime}(\mu) Y\right]=0 .
$$

If $g$ is a convex function, it follows that

$$
\frac{\partial^{2} f}{\partial \sigma^{2}}(\mu, \sigma)=E\left[g^{\prime \prime}(\mu+\sigma Y) Y^{2}\right] \geq 0 .
$$

Putting these two results together gives

$$
\frac{\partial f}{\partial \sigma}(\mu, \sigma) \geq 0
$$

so that $E[g(X)]=f(\mu, \sigma)$ is indeed increasing in volatility. The proof for a concave function $g$ proceeds analogously.

\section{A.2 Expected option price after a jump}

In Section 4.2 we decompose the expected excess return of an option into different components. To do so, we have - among other things - to calculate the expected call price after a jump in the stock price

$$
E\left[C\left(S e^{X}, V\right)\right]=\int_{-\infty}^{\infty} C\left(S e^{x}, V\right) f(x) d x
$$

where $f$ denotes the density of the jump size $x$. The integration from $-\infty$ to $\infty$ leads to some numerical problems, since the call price is nearly zero for low stock prices and cannot be calculated precisely any more, while the probability of reaching these low stock prices is too large to be neglected.

We assume that we can calculate the call price for $x \geq k$, but run into numerical problems for $x<k$. The upper and lower bounds for the expected call price after a jump occurred are

$$
E\left[C\left(S e^{X}, V\right)\right] \geq \int_{k}^{\infty} C\left(S e^{x}, V\right) f(x) d x+0 \int_{-\infty}^{k} 0 f(x) d x
$$

and

$$
E\left[C\left(S e^{X}, V\right)\right] \leq \int_{k}^{\infty} C\left(S e^{x}, V\right) f(x) d x+\int_{-\infty}^{k} C\left(S e^{k}, V\right) f(x) d x
$$

The two bounds are nearly indistinguishable. In a similar way, we can calculate the expected call price after a simultaneous jump in the stock price and in volatility. 


\section{A.3 Stationary distribution of $\mathrm{V}$}

The numerical results for the decomposition of the expected local returns in Section 4.2 are based on the long run averages, and we integrate volatility out, using the stationary distribution of volatility.

Conditional on $V_{t}$, the volatility $V_{T}$ in the SVJ model is distributed as $e^{-\kappa(T-t)} / n(t, T)$ times a non-central chi-squared distribution with $d$ degrees of freedom and noncentrality parameter $V_{t} \cdot n(t, T)$ :

$$
\operatorname{Pr}\left(V_{T}<x \mid V_{t}\right)=F_{\chi^{\prime 2}}\left(\frac{x \cdot n(t, T)}{e^{-\kappa(T-t)}} ; d, V_{t} \cdot n(t, T)\right)
$$

where

$$
\begin{aligned}
d & =\frac{4 \kappa \theta}{\sigma_{V}^{2}} \\
n(t, T) & =\frac{4 \kappa e^{-\kappa(T-t)}}{\sigma_{V}^{2}\left(1-e^{-\kappa(T-t)}\right)} .
\end{aligned}
$$

The probablity density function for $V$ is therefore given by

$$
f_{V_{T} \mid V_{t}}(x)=f_{\chi^{\prime 2}}\left(\frac{x \cdot n(t, T)}{e^{-\kappa(T-t)}} ; d, V_{t} \cdot n(t, T)\right) \cdot \frac{n(t, T)}{e^{-\kappa(T-t)}} .
$$

For $T \rightarrow \infty$, we get for the stationary distribution of $V$, which is given by

$$
f_{V}(x)=f_{\chi^{2}}\left(\frac{x \cdot 4 \kappa}{\sigma_{V}^{2}} ; d\right) \cdot \frac{4 \kappa}{\sigma_{V}^{2}}
$$

since

$$
\begin{aligned}
& \lim _{T \rightarrow \infty} n(0, T)=0 \\
& \lim _{T \rightarrow \infty} \frac{n(0, T)}{e^{-\kappa T}}=\frac{4 \kappa}{\sigma_{V}^{2}}
\end{aligned}
$$

For more details, see Andersen (2007) and the references therein.

\section{A.4 Density under $\mathbb{P}$ and under $\mathbb{Q}$}

The density under the physical as well as under the risk-neutral measure can calculated by Fourier inversion. We denote the density of the logarithm of the stock at time $\mathrm{T}$ by $f_{\ln S_{T}}$. It is given by

$$
f_{\ln S_{T}}(\ln x, t, T, s, v)=\frac{1}{2 \pi} \int_{-\infty}^{\infty} R e\left[e^{-i u \ln x} \phi(u, t, T, s, v)\right] d u .
$$


The function $\phi$ is the Fourier transform and defined by

$$
\phi\left(u, t, T, S_{t}, V_{t}\right)=E^{\mathbb{P}}\left[e^{i u \ln S_{T}} \mid \mathcal{F}_{t}\right] .
$$

Solutions for this transform are well known for the affine models we consider in the paper and can be found in the literature (see, e.g., Bakshi, Cao, and Chen (1997) or Duffie, Pan, and Singleton (2000)). The density of the stock at time T then follows from

$$
f_{S_{T}}(x, t, T, s, v)=f_{\ln S_{T}}(\ln x, t, T, s, v) \frac{1}{x} .
$$

Figures 10 and 11 show the densities for $S$ when $V_{t}=\theta^{\mathbb{P}}$. As a robustness check, we also calculated the long run average of the density by integrating out the current local volatility, using the stationary distribution of $V$. The results stay essentially unchanged.

\section{B Robustness Check}

\section{B.1 Differences in the Distributions of Option Returns}

Expected option returns depend on the model and on the exact specification of risk premia. We now test whether the differences between the return distributions generated by the various models in Section 4.1 are also statistically significant. If this is the case, observed options returns might help in identifying the structure of (jump) risk premia.

We use the Kolmogorov-Smirnov test to test whether two distributions (generated by the same model, but with different restrictions on the risk premia) come from the same true continuous distribution or not. The t-test is used to test for equality of means between two distributions. It can be applied in our setup due to the central limit theorem.

The results for the SVJ model show that the distributions and means of option returns are statistically different from each other for combinations of restricted 
versions of the model. This holds true for nearly all moneyness categories and for intermediate as well as maturity returns. For the SVCJ model, the same result holds true for intermediate and maturity call returns and for intermediate put returns. For maturity put returns, however, the Kolmogorov-Smirnov test cannot reject the null hypothesis that the SVCJ model without restrictions and the two versions with $\sigma_{S}^{\mathbb{Q}}=\sigma_{S}^{\mathbb{P}}$ come from the same continuous distribution. The t-test still rejects the hypothesis that these distributions have the same mean. Therefore, we are not able to distinguish different restrictions on the risk premia from the distributions of maturity put returns in the SVCJ model. However, we are able to do so with intermediate put returns as well as call returns. 


\section{References}

Andersen, L., 2007, Efficient Simulation of the Heston Stochastic Volatility Model, Working Paper.

Bakshi, G., C. Cao, and Z. Chen, 1997, Empirical Performance of Alternative Option Pricing Models, Journal of Finance 52, 2003-2049.

Bakshi, G., and N. Kapadia, 2003, Delta-Hedged Gains and the Negative Market Volatility Risk Premium, Review of Financial Studies 16, 527-566.

Bates, D.S., 1988, Pricing options under jump-diffusion processes, Working Paper, University of Pennsylvania.

Bates, D.S., 1996, The Skewness Premium: Option Pricing Under Asymmetric Information, Working Paper.

Bates, D.S., 2000, Post-'87 Crash Fears in the S\&P Futures Option Market, Journal of Econometrics 94, 181-238.

Benzoni, L., 2002, Pricing Options under Stochastic Volatility: An Empirical Investigation, Working Paper.

Bollen, N.P.P., and R.E. Whaley, 2004, Does Net Buying Pressure Affect the Shape of Implied Volatility Functions?, Journal of Finance 59, 711-753.

Bondarenko, O., 2003, Why are Put Options So Expensive?, Working Paper.

Broadie, M., M. Chernov, and M. Johannes, 2007, Model Specification and Risk Premia: Evidence from Futures Options, Journal of Finance 62, 1453-1490.

Broadie, M., M. Chernov, and M. Johannes, 2008, Understanding Index Option Returns, Working Paper.

Buraschi, A., and J. Jackwerth, 2001, The Price of a Smile: Hedging and Spanning in Option Markets, Review of Financial Studies 14, 495-527. 
Chernov, M., and E. Ghysels, 2000, A Study towards a Unified Approach to the Joint Estimation of Objective and Risk Neutral Measures for the Purpose of Option Pricing, Journal of Financial Economics 56, 407-458.

Coval, J.D., and T. Shumway, 2001, Expected Options Returns, Journal of Finance 56, 983-1009.

Driessen, J., and P. Maenhout, 2007, An Empirical Portfolio Perspective on Option Pricing Anomalies, Working Paper.

Duffie, D., J. Pan, and K. Singleton, 2000, Transform Analysis and Asset Pricing for Affine Jump Diffusions, Econometrica 68, 1343-1376.

Eraker, B., M. Johannes, and N. Polson, 2003, The Impact of Jumps in Volatility and Returns, Journal of Finance 58, 1269-1300.

Heston, S.L., 1993, A Closed-Form Solution for Options with Stochastic Volatility with Applications to Bond and Currency Options, Review of Financial Studies 6, $327-343$.

Ibánez, A., 2007, On the Negative Market Volatilty Risk-Premium: Bridging the Gap Between Option Returns and the Pricing of Options, Working Paper.

Jones, C.S., 2003, The dynamics of stochastic volatility: Evidence from underlying and options markets, Journal of Econometrics 116, 181-224.

Jones, C.S., 2006, A Nonlinear Factor Analysis of S\&P 500 Option Returns, Journal of Finance 61, 2325-2363.

Merton, R.C., 1976, Option Pricing When Underlying Stock Returns are Discontinuous, Journal of Financial Economics 3, 125-144.

Naik, V., and M. Lee, 1990, General equilibrium pricing of options on the market portfolio with discontinuous returns, Review of Financial Studies 3, 493-521.

Pan, J., 2002, The Jump-Risk Premia Implicit in Options: Evidence from an Integrated Time-Series Study, Journal of Financial Economics 63, 3-50. 


\begin{tabular}{|c||c|c|c|c||c|c|c|c|}
\hline \multicolumn{1}{|c||}{ Param. } & \multicolumn{9}{c||}{ option-based parameterization } & \multicolumn{5}{c|}{ time-series based parameterization } \\
& BS & SV & SVJ & SVCJ & BS & SJ & SV & SVJ \\
\hline \hline$r$ & 0.045 & 0.045 & 0.045 & 0.045 & 0.045 & 0.045 & 0.045 & 0.045 \\
\hline$a$ & & & & & 0.054 & 0.054 & 0.054 & 0.054 \\
\hline$b$ & 0.1119 & 0.1119 & 0.1250 & 0.1396 & & & & \\
\hline$\kappa^{\mathbb{P}}$ & & 5.8212 & 3.2256 & 6.5520 & & & 5.33 & 5.33 \\
\hline$\theta^{\mathbb{P}}$ & 0.0228 & 0.0228 & 0.0205 & 0.0135 & 0.0225 & 0.0183 & 0.0225 & 0.0183 \\
\hline$\sigma_{V}$ & & 0.3614 & 0.2404 & 0.1991 & & & 0.14 & 0.14 \\
\hline$\rho$ & & -0.3974 & -0.4668 & -0.4838 & & & -0.52 & -0.52 \\
\hline$\mu_{V}^{\mathbb{P}}$ & & & & 0.0374 & & & & \\
\hline$\mu_{S}^{\mathbb{P}}$ & & & -0.0259 & -0.0175 & & -0.0325 & & -0.0325 \\
\hline$\rho_{J}^{\mathbb{P}}$ & & & & -0.2384 & & & & \\
\hline$\sigma_{S}^{\mathbb{P}}$ & & & 0.0407 & 0.0289 & & 0.060 & & 0.060 \\
\hline$\lambda^{\mathbb{P}}$ & & & 1.5120 & 1.6632 & & 0.91 & & 0.91 \\
\hline
\end{tabular}

Table 1: Parameters under the $\mathbb{P}$-measure

The parameters under the physical measure are taken from Eraker, Johannes, and Polson (2003) for the option-based parameterization and from Broadie, Chernov, and Johannes (2008) for the time-series based parameterization. All parameters are given as annual decimals. 


\begin{tabular}{|c|c|c|c|c|c|c|c|}
\hline \multicolumn{2}{|c|}{ Parameters } & restriction & $\eta_{V}$ & $\mu_{S}^{\mathbb{Q}}$ & $\sigma_{S}^{\mathbb{Q}}$ & $\mu_{V}^{\mathbb{Q}}$ & $\lambda^{\mathbb{Q}}$ \\
\hline \multirow{9}{*}{$\begin{array}{c}\text { option-based } \\
\text { parameterization }\end{array}$} & SV & & 1.26 & & & & \\
\hline & SVJ & & 1.512 & -0.0491 & 0.0994 & & $\lambda^{\mathbb{P}(*)}$ \\
\hline & SVJ & $\eta_{V}=0$ & $0^{(*)}$ & -0.0482 & 0.0981 & & $\lambda^{\mathbb{P}(*)}$ \\
\hline & SVJ & $\sigma_{S}^{Q}=\sigma_{S}^{P}$ & 2.52 & -0.0997 & $\sigma_{S}^{\mathbb{P}(*)}$ & & $\lambda^{\mathbb{P}(*)}$ \\
\hline & SVJ & $\eta_{V}=0, \sigma_{S}^{Q}=\sigma_{S}^{P}$ & $0^{(*)}$ & -0.0969 & $\sigma_{S}^{\mathbb{P}(*)}$ & & $\lambda^{\mathbb{P}(*)}$ \\
\hline & SVCJ & & 7.812 & -0.0539 & 0.0578 & 0.2213 & $\lambda^{\mathbb{P}(*)}$ \\
\hline & SVCJ & $\eta_{V}=0$ & $0^{(*)}$ & -0.0501 & 0.0751 & 0.0935 & $\lambda^{\mathbb{P}(*)}$ \\
\hline & SVCJ & $\sigma_{S}^{Q}=\sigma_{S}^{P}$ & 7.56 & -0.0658 & $\sigma_{S}^{\mathbb{P}(*)}$ & 0.2724 & $\lambda^{\mathbb{P}(*)}$ \\
\hline & SVCJ & $\eta_{V}=0, \sigma_{S}^{Q}=\sigma_{S}^{P}$ & $0^{(*)}$ & -0.0725 & $\sigma_{S}^{\mathbb{P}(*)}$ & 0.1333 & $\lambda^{\mathbb{P}(*)}$ \\
\hline \multirow{2}{*}{$\begin{array}{l}\text { time-series } \\
\text { based param. }\end{array}$} & SJ & & $0^{(*)}$ & -0.0685 & $\sigma_{S}^{\mathbb{P}(*)}$ & & 1.51 \\
\hline & SVJ & & $0^{(*)}$ & -0.0685 & $\sigma_{S}^{\mathbb{P}(*)}$ & & 1.51 \\
\hline
\end{tabular}

Table 2: Parameters under the $\mathbb{Q}$-measure

The parameters under the risk-neutral measure are taken from Broadie, Chernov, and Johannes (2007) for the option-based parameterization and from Broadie, Chernov, and Johannes (2008) for the time-series based parameterization. All parameters are given as annual decimals.

$\left.{ }^{*}\right)$ by assumption 


\begin{tabular}{|c||c|c|c|c||c|c||c|c|c|c|}
\hline \multicolumn{1}{|c||}{} & \multicolumn{9}{c||}{ time-series based parameterization } & \multicolumn{3}{c|}{ option-based parameterization } \\
M & BS & SJ & SV & SVJ & SJR & SVJR & BS & SV & SVJ & SVCJ \\
\hline \hline 96 & 44.97 & 43.47 & 44.00 & 43.04 & 26.14 & 25.79 & 21.15 & 13.21 & 19.70 & 30.91 \\
\hline 98 & 29.52 & 27.13 & 27.68 & 25.84 & 38.70 & 38.58 & 11.45 & 4.54 & 32.86 & 46.62 \\
\hline 100 & 45.18 & 42.36 & 42.28 & 40.01 & 20.26 & 19.52 & 22.34 & 7.73 & 15.38 & 42.64 \\
\hline 102 & 19.62 & 21.26 & 21.77 & 23.56 & 2.78 & 2.31 & 40.06 & 27.60 & 1.03 & 14.58 \\
\hline
\end{tabular}

Table 3: P-values for intermediate monthly call returns in $\%$

The table shows the p-values for intermediate monthly call returns related to the observed data of Coval and Shumway (2001) for different specifications and models (see Section 2.1). For details about p-values see Section 2.2. 


\begin{tabular}{|c||c|c|c|c||c|c||c|c|c|c|}
\hline \multicolumn{1}{|c||}{} & \multicolumn{4}{c||}{ time-series based parameterization } & \multicolumn{5}{c|}{ option-based parameterization } \\
M & BS & SJ & SV & SVJ & SJR & SVJR & BS & SV & SVJ & SVCJ \\
\hline \hline 96 & 0.00 & 0.00 & 0.00 & 0.00 & 0.00 & 0.00 & 0.00 & 0.00 & 0.00 & 0.01 \\
\hline 98 & 0.00 & 0.00 & 0.00 & 0.00 & 0.00 & 0.00 & 0.00 & 0.00 & 0.00 & 0.00 \\
\hline 100 & 0.10 & 0.17 & 0.15 & 0.22 & 0.61 & 0.87 & 0.63 & 0.63 & 0.96 & 1.04 \\
\hline 102 & 0.20 & 0.26 & 0.26 & 0.35 & 0.66 & 0.95 & 1.11 & 1.29 & 1.07 & 1.04 \\
\hline
\end{tabular}

Table 4: P-values for intermediate monthly put returns in $\%$

The table shows the p-values for intermediate monthly put returns related to the observed data of Coval and Shumway (2001) for different specifications and models (see Section 2.1). For details about p-values see Section 2.2. 


\begin{tabular}{|c||c|c|c|c||c|c||c|c|c|c|}
\hline \multicolumn{1}{|c||}{} & \multicolumn{9}{c||}{ time-series based parameterization } & \multicolumn{3}{c|}{ option-based parameterization } \\
M & BS & SJ & SV & SVJ & SJR & SVJR & BS & SV & SVJ & SVCJ \\
\hline \hline 94 & 11.92 & 12.28 & 11.21 & 10.95 & 33.64 & 40.49 & 16.53 & 16.10 & 26.19 & 33.90 \\
\hline 96 & 3.00 & 4.61 & 3.15 & 4.47 & 43.60 & 39.20 & 5.94 & 6.56 & 48.07 & 41.60 \\
\hline 98 & 0.79 & 1.61 & 1.09 & 1.81 & 18.74 & 19.60 & 2.64 & 3.56 & 26.04 & 19.55 \\
\hline 100 & 2.80 & 4.05 & 3.45 & 4.76 & 20.34 & 22.59 & 8.66 & 9.96 & 29.54 & 20.14 \\
\hline 102 & 9.74 & 11.55 & 10.70 & 12.64 & 25.76 & 27.78 & 24.51 & 27.10 & 35.24 & 26.58 \\
\hline
\end{tabular}

Table 5: P-values for monthly maturity put returns in \%

The table shows the p-values for monthly maturity put returns related to the observed data of Broadie, Chernov, and Johannes (2008) for different specifications and models (see Section 2.1). For details about p-values see Section 2.2.

Remark: Broadie, Chernov, and Johannes (2008) define their p-values slightly different. They report as p-values the probability of obtaining a result that is smaller then the respective observed returns. 
Panel A

\begin{tabular}{|c|c||c|c||c|c|c|c|}
\hline \multirow{2}{*}{ Model } & restriction & $\eta_{S}$ & $\eta_{V}$ & call return & $E R^{S}$ & $E R^{V}$ & $E R^{J}$ \\
\hline \hline \multirow{2}{*}{$\mathrm{SV}$} & & 3.4342 & 1.26 & + & + & + & 0 \\
\cline { 2 - 8 } & $\eta_{V}=0$ & 3.4342 & 0 & + & + & 0 & 0 \\
\hline \hline \multirow{2}{*}{ SVJ } & & 1.2163 & 1.512 & - for $K \geq 106$ & + & + & - for $K \geq 94$ \\
\cline { 2 - 8 } & $\eta_{V}=0$ & 1.2707 & 0 & - for $K \geq 102$ & + & 0 & - for $K \geq 94$ \\
\cline { 2 - 8 } & $\sigma_{S}^{Q}=\sigma_{S}^{P}$ & -2.5410 & 2.52 & + & - & + & + \\
\cline { 2 - 8 } & $\eta_{V}=0, \sigma_{S}^{Q}=\sigma_{S}^{P}$ & -2.3537 & 0 & - for $K \geq 100$ & - & 0 & + \\
\hline \hline \multirow{3}{*}{ SVCJ } & & 1.2379 & 7.812 & - for $K \geq 108$ & + & + & - for $K \geq 94$ \\
\cline { 2 - 8 } & $\eta_{V}=0$ & 1.8181 & 0 & - for $K \geq 100$ & + & 0 & - for $K \geq 94$ \\
\cline { 2 - 8 } & $\sigma_{S}^{Q}=\sigma_{S}^{P}$ & -0.2900 & 7.56 & - for $K \geq 108$ & - & + & - for $K \geq 98$ \\
\cline { 2 - 8 } & $\eta_{V}=0, \sigma_{S}^{Q}=\sigma_{S}^{P}$ & -1.0606 & 0 & - for $K \geq 98$ & - & 0 & - for $K \geq 104$ \\
\hline
\end{tabular}

Panel B

\begin{tabular}{|c|c||c||c|c|c|c|}
\hline Model & restriction & $E R^{J}$ & $E R_{\lambda}^{J}$ & $E R_{\mu}^{J}$ & $E R_{\sigma}^{J}$ & $E R_{V}^{J}$ \\
\hline \hline \multirow{3}{*}{ SV } & & 0 & 0 & 0 & 0 & 0 \\
\cline { 2 - 7 } & $\eta_{V}=0$ & 0 & 0 & 0 & 0 & 0 \\
\hline \hline \multirow{3}{*}{ SVJ } & & - for $K \geq 94$ & 0 & + & - & 0 \\
\cline { 2 - 7 } & $\eta_{V}=0$ & - for $K \geq 94$ & 0 & + & - & 0 \\
\cline { 2 - 7 } & $\sigma_{S}^{Q}=\sigma_{S}^{P}$ & + & 0 & + & 0 & 0 \\
\cline { 2 - 7 } & $\eta_{V}=0, \sigma_{S}^{Q}=\sigma_{S}^{P}$ & + & 0 & + & 0 & 0 \\
\hline \hline \multirow{3}{*}{ SVCJ } & $\eta_{V}=0$ & - for $K \geq 94$ & 0 & + & - & - \\
\cline { 2 - 7 } & $\sigma_{S}^{Q}=\sigma_{S}^{P}$ & - for $K \geq 94$ & 0 & + & - & - \\
\cline { 2 - 7 } & $\eta_{V}=0, \sigma_{S}^{Q}=\sigma_{S}^{P}$ & - for $K \geq 98$ & 0 & + & 0 & - \\
\cline { 2 - 7 } & & + & 0 & + & 0 & - \\
\hline
\end{tabular}

Table 6: Risk premia and decomposition of local call returns

The table gives the risk premia for stock diffusion risk and volatility diffusion risk in the option-based parametrization for several restrictions on the risk premia.

Panel A shows the contribution of the market prices of risk for stock diffusion risk, volatility diffusion risk, and jump risk to the expected option return. Column 5 gives the sign of the intermediate mean call return. Columns 6 to 8 give the sign of the contributions to the local expected call returns.

Panel B shows the contribution of the market prices of risk for jump intensity risk, jump size risk, jump variance risk, and variance jump risk to the overall jump risk premium in option returns. 

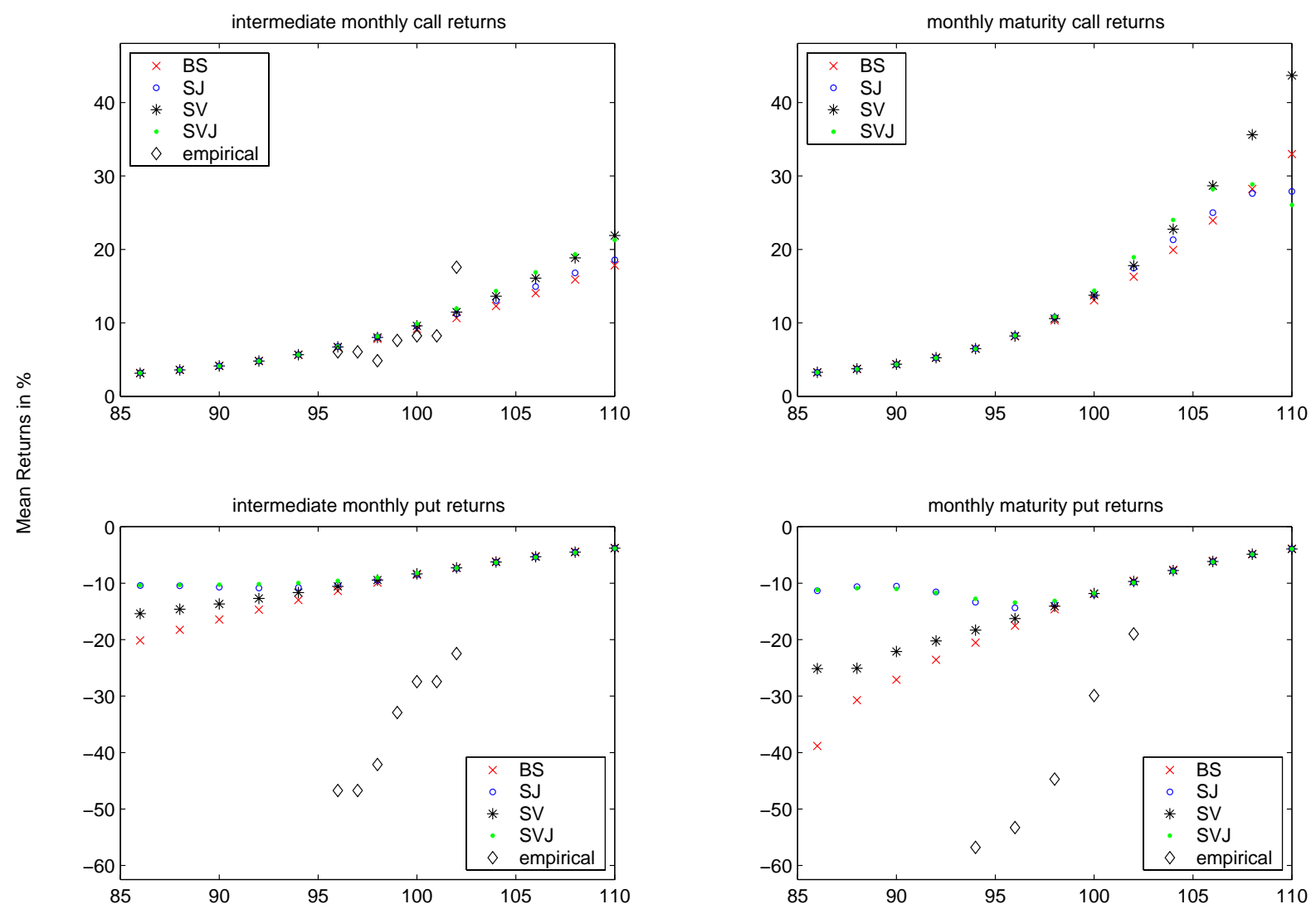

Moneyness in \%

Figure 1: Models under time-series based specification with equity premium only The graphs show the simulated intermediate and maturity call and put returns for a holding period of one month and for all models. The parameters are taken from the time-series based specification with a premium for stock diffusion risk only (see Section 3.1). As a comparison we display empirical intermediate and maturity returns from Coval and Shumway (2001) and Broadie, Chernov, and Johannes (2008), respectively. 

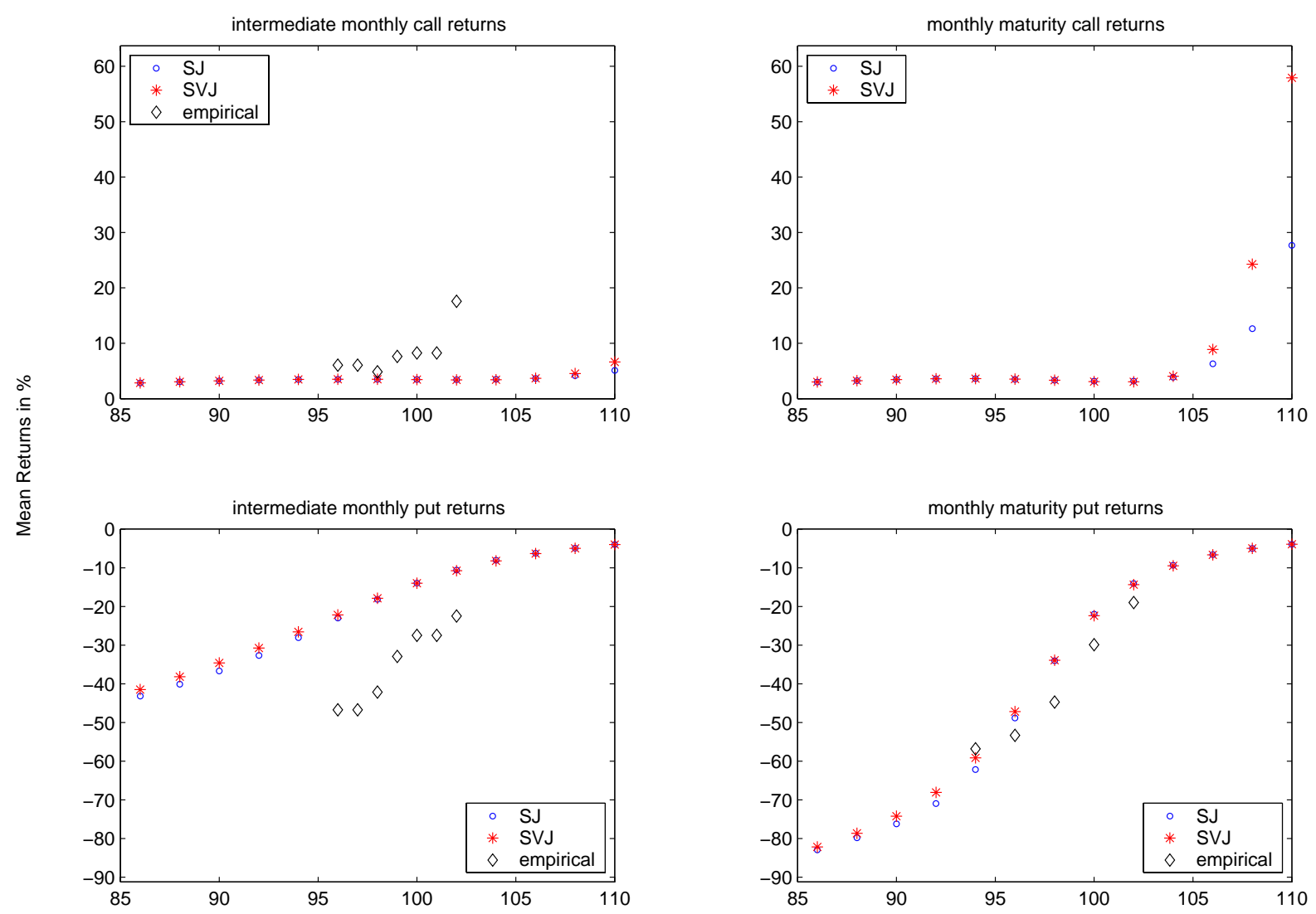

Moneyness in \%

Figure 2: Models under time-series based specification with jump risk premia The graphs show the simulated intermediate and maturity call and put returns for a holding period of one month and for all models. The parameters are taken from the time-series based specification where stock diffusion risk and stock jump risk are priced (see Section 3.2). As a comparison we display the empirical intermediate and maturity returns from Coval and Shumway (2001) and Broadie, Chernov, and Johannes (2008), respectively. 

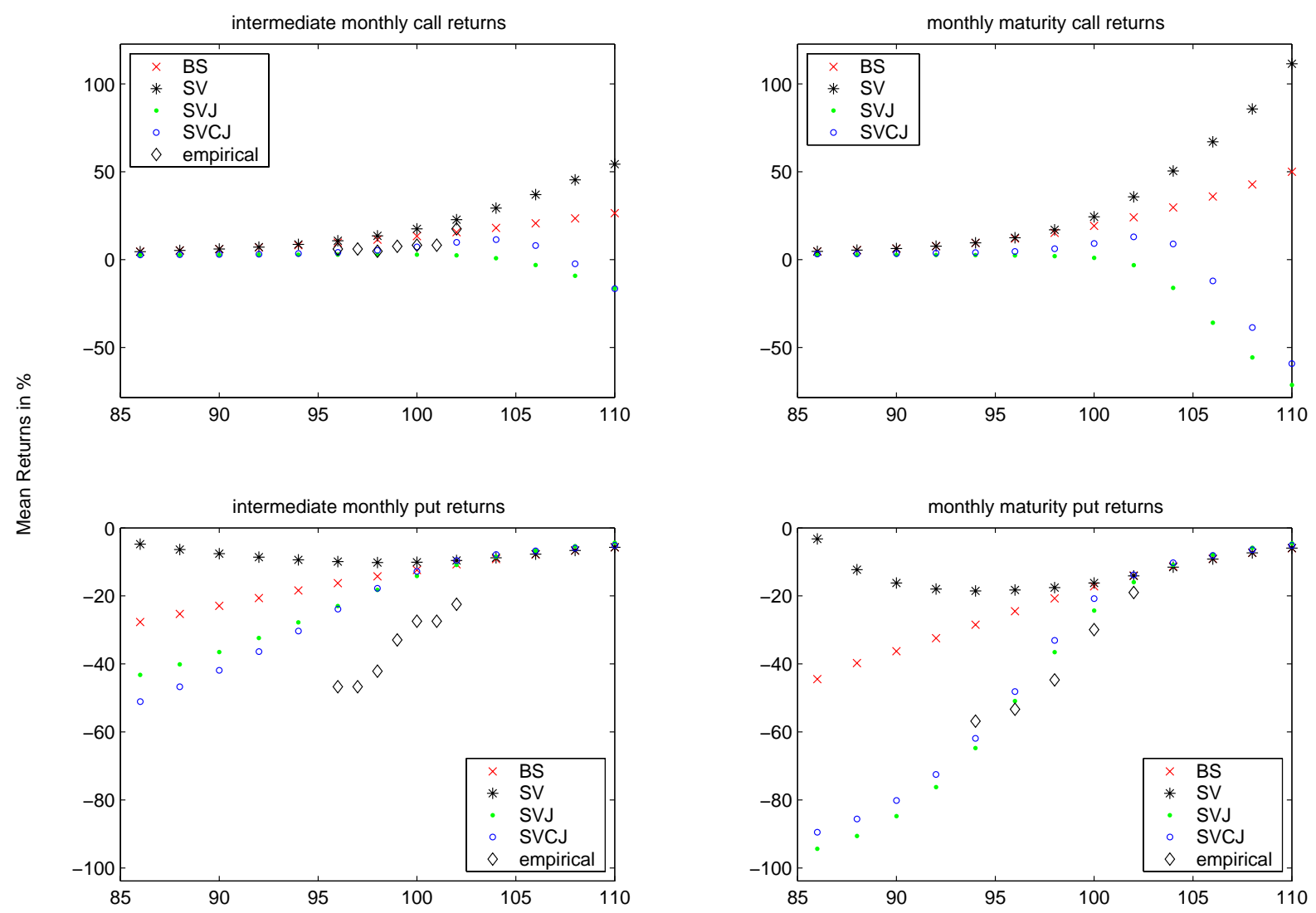

Moneyness in \%

Figure 3: Models under option-based specification without constraints

The graphs show the simulated intermediate and maturity call and put returns for a holding period of one month and for all models. The parameters are taken from the option-based specification. As a comparison we display the empirical intermediate and maturity returns as published in Coval and Shumway (2001) and Broadie, Chernov, and Johannes (2008), respectively. 

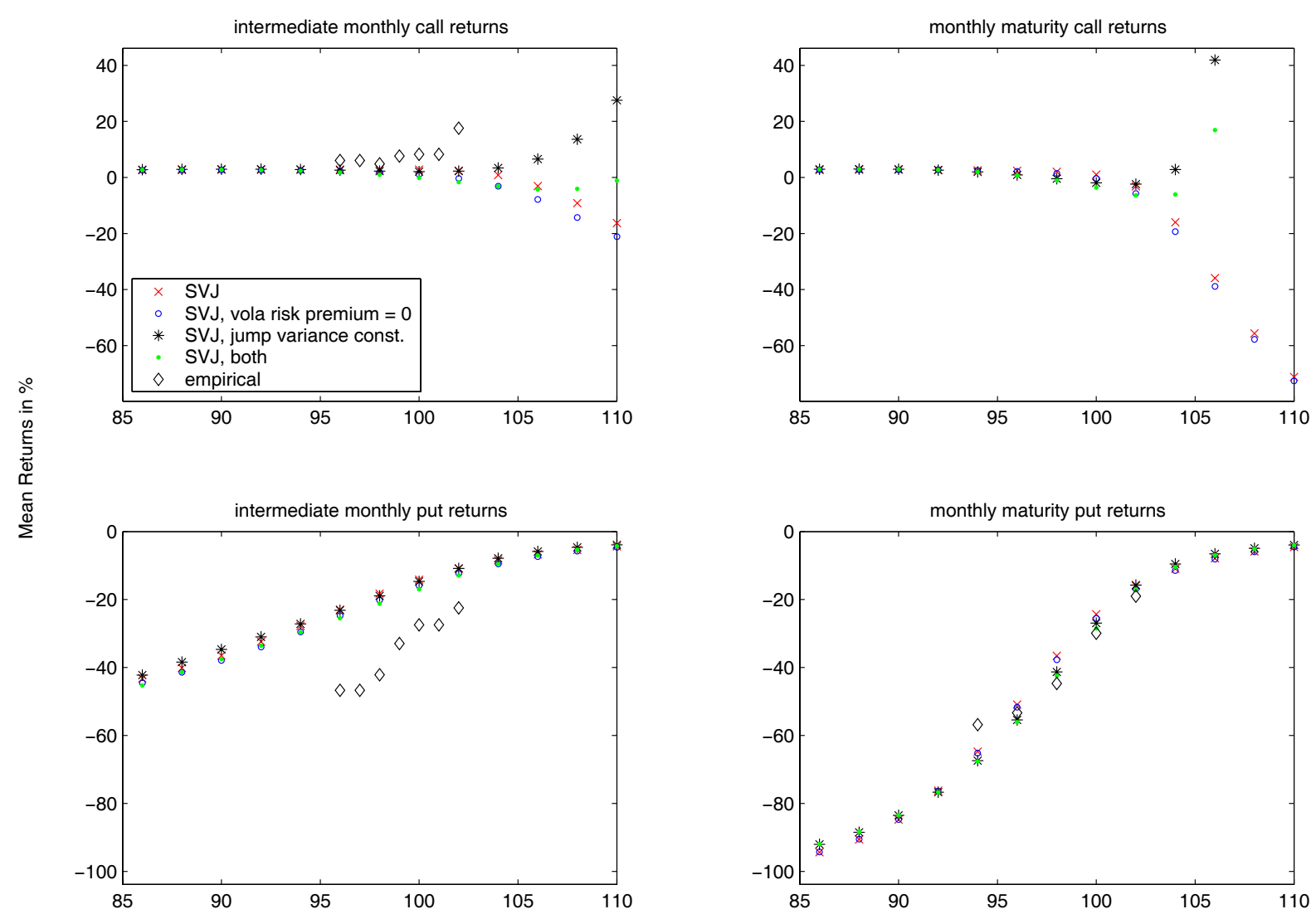

Moneyness in \%

Figure 4: SVJ under option-based specification with restrictions

The graphs show the simulated intermediate and maturity call and put returns for a holding period of one month for the SVJ model. The parameters are taken from the option-based specification where the premium on jump variance risk and/or volatility risk is restricted to be equal to zero, as described in Section 4.2. As a comparison we display the empirical intermediate and maturity returns as published in Coval and Shumway (2001) and Broadie, Chernov, and Johannes (2008), respectively. 

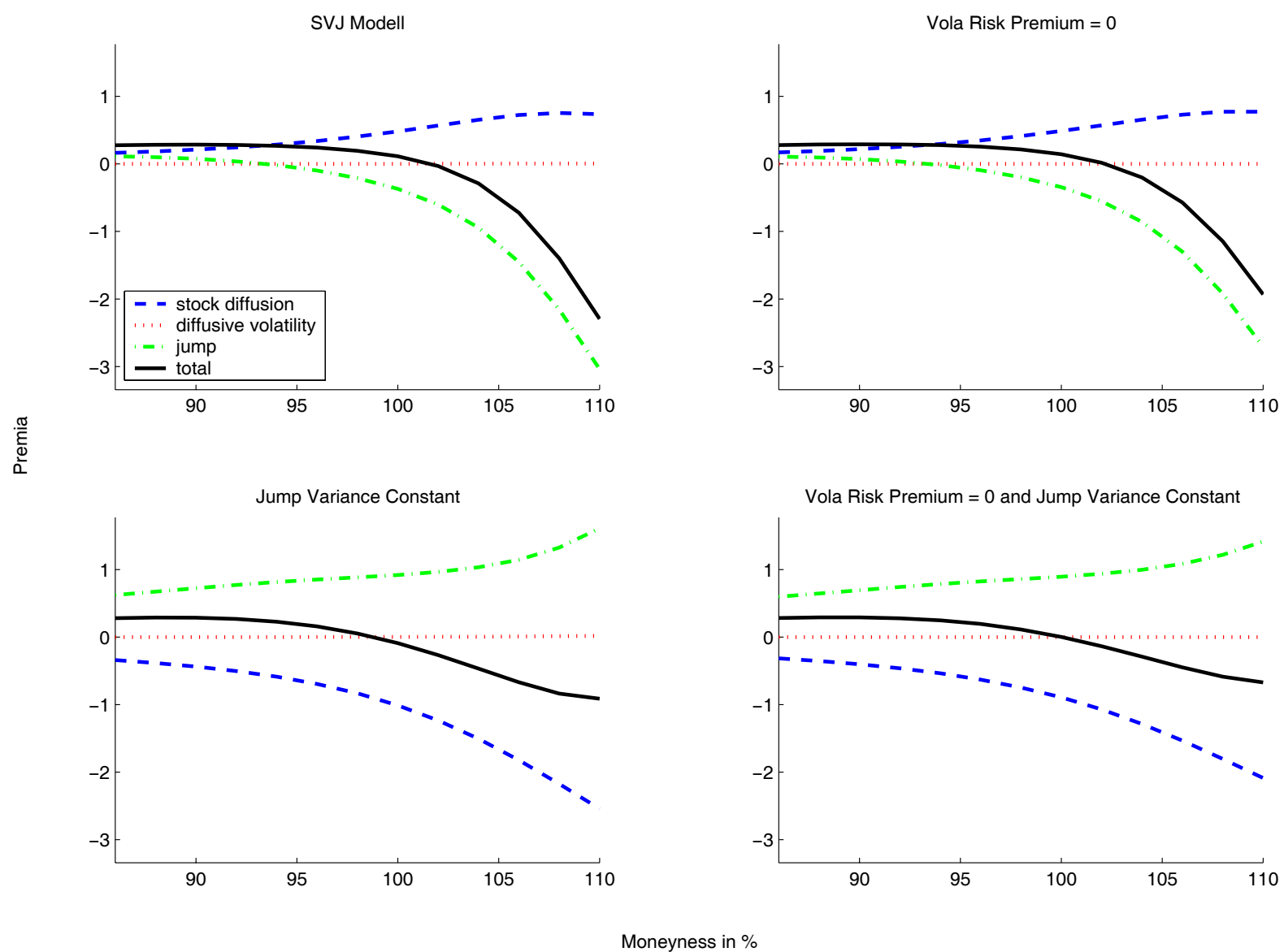

Figure 5: Decomposition of local expected return for the SVJ Model

The graphs show the decomposition of the local expected returns in the SVJ model. Details about the decomposition can be found in 4.2. The parameters are taken from the option-based specification where the premium on jump variance risk and/or volatility risk is restricted to be equal to zero, as described in Section 4.2. 

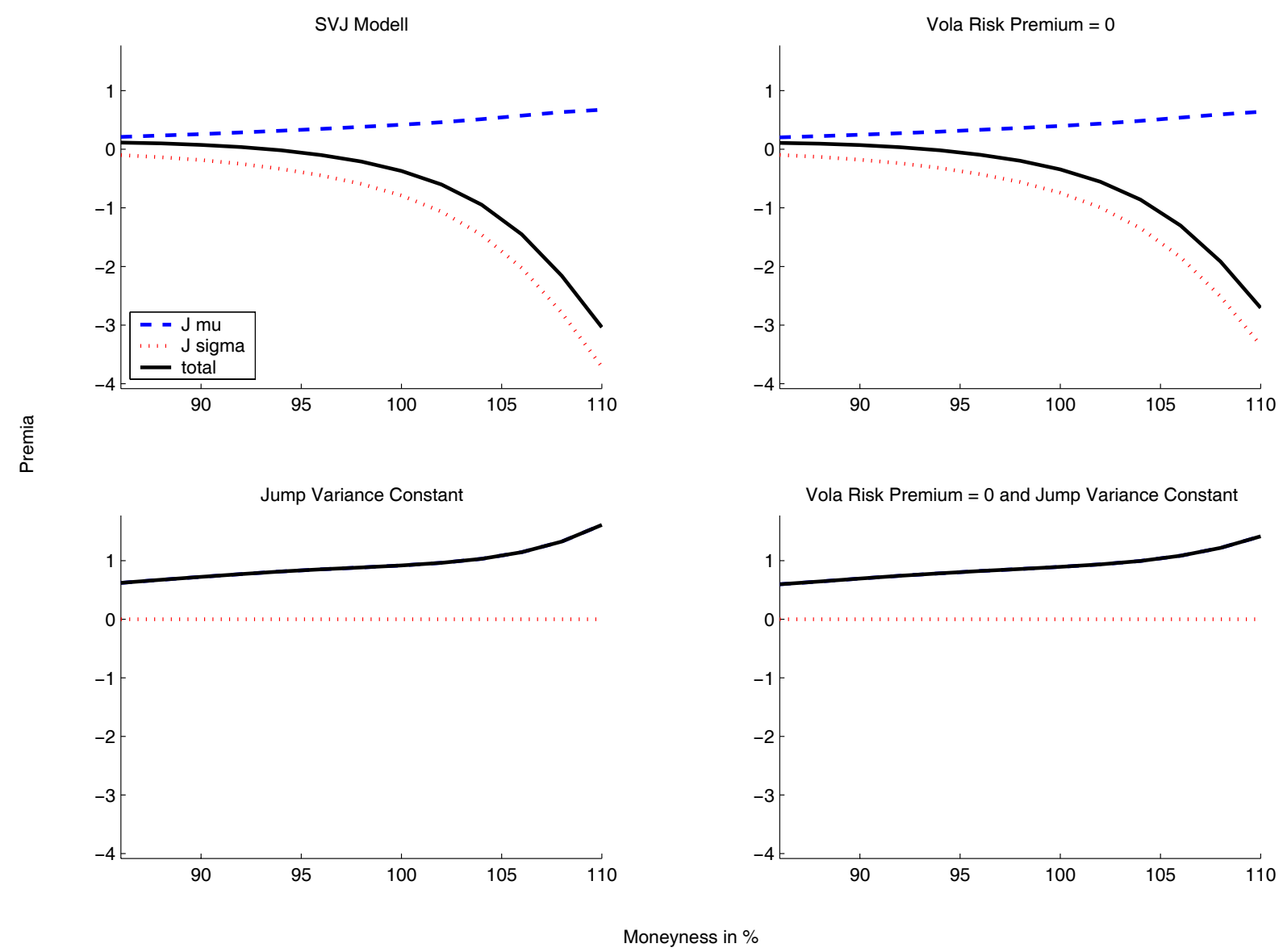

Figure 6: Decomposition of local expected jump risk premia for the SVJ Model The graphs show the decomposition of the local expected jump risk premia in the SVJ model. Details about the decomposition can be found in 4.2. The parameters are taken from the option-based specification where the premium on jump variance risk and/or volatility risk is restricted to be equal to zero, as described in Section 4.2 . 

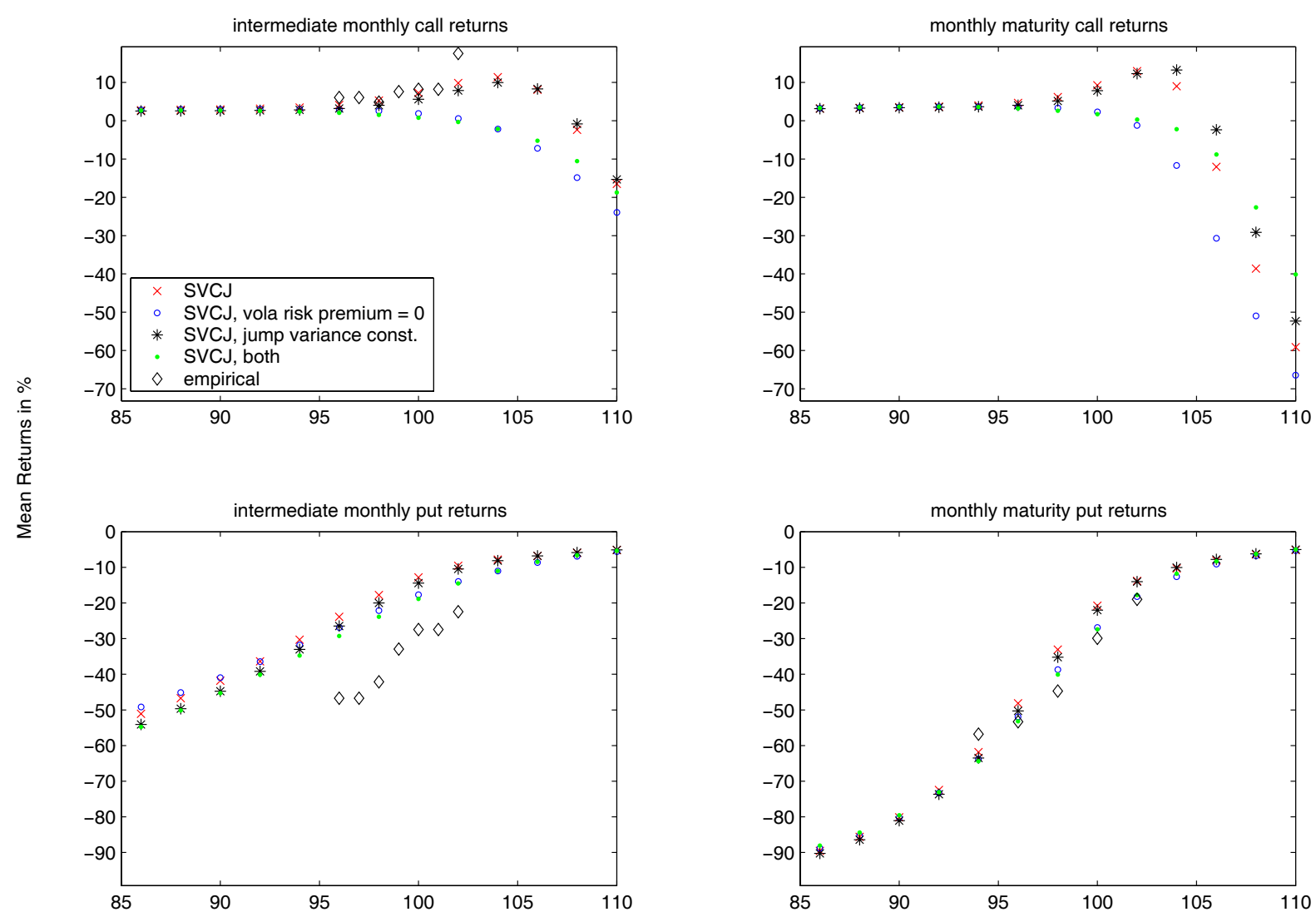

Moneyness in \%

Figure 7: SVCJ under option-based specification with restrictions

The graphs show the simulated intermediate and maturity call and put returns for a holding period of one month for the SVCJ model. The parameters are taken from the option-based specification where the premium on jump variance risk and/or volatility risk is restricted to be equal to zero, as described in Section 4.2. As a comparison we display the empirical intermediate and maturity returns as published in Coval and Shumway (2001) and Broadie, Chernov, and Johannes (2008), respectively. 

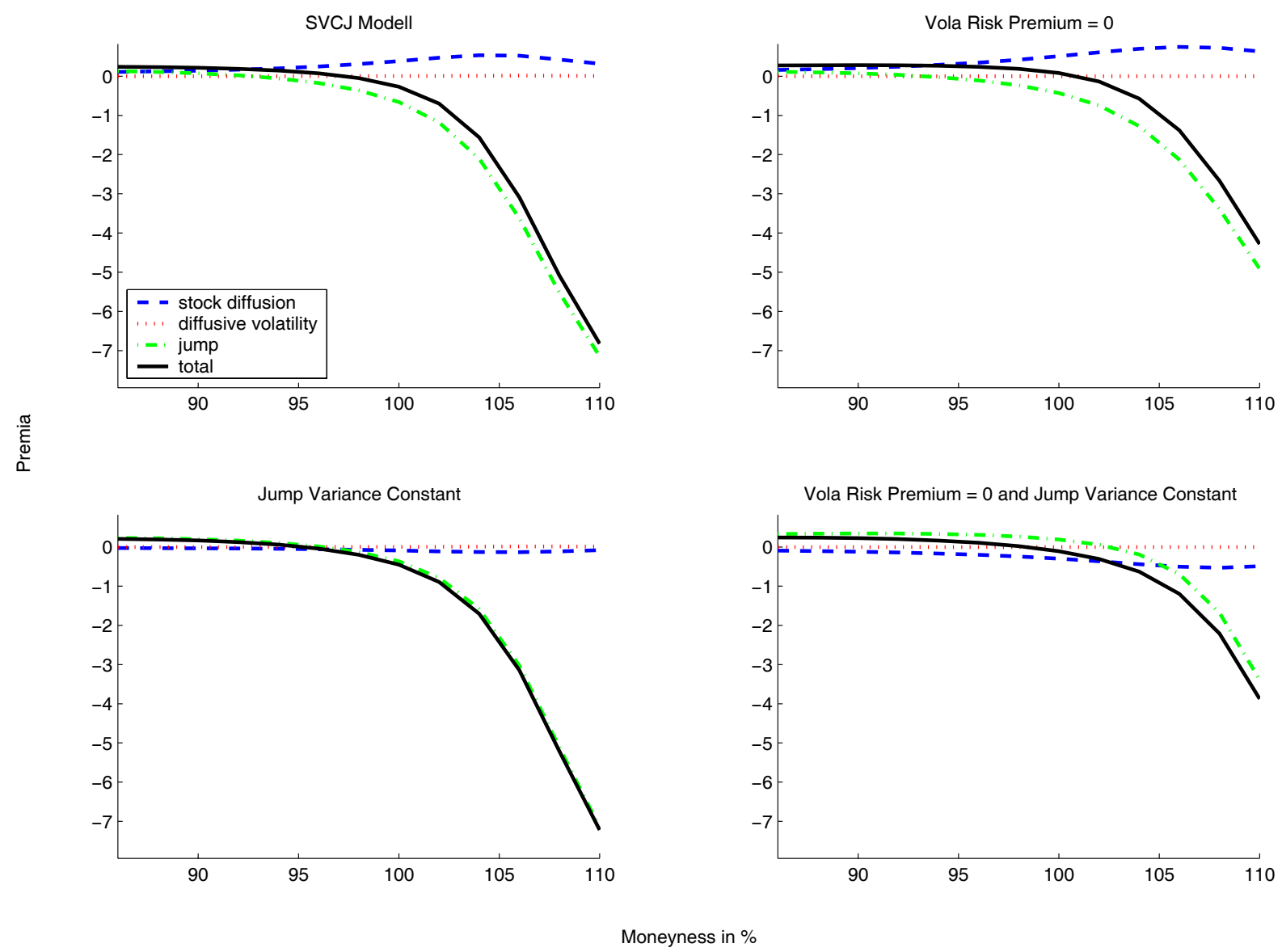

Figure 8: Decomposition of local expected return for the SVCJ Model

The graphs show the decomposition of the local expected returns in the SVCJ model. Details about the decomposition can be found in 4.2. The parameters are taken from the option-based specification where the premium on jump variance risk and/or volatility risk is restricted to be equal to zero, as described in Section 4.2. 

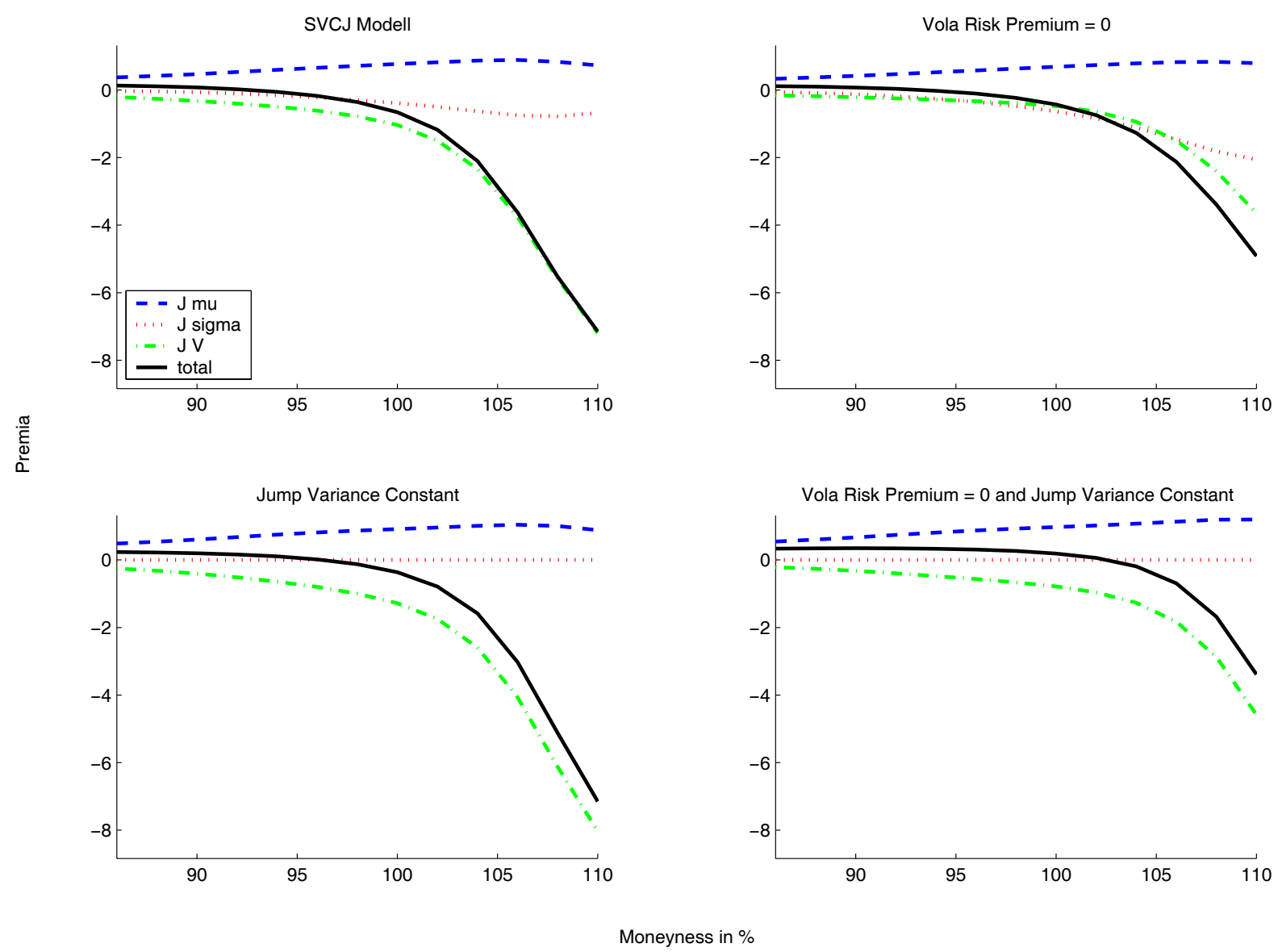

Figure 9: Decomposition of local expected jump risk premia for the SVCJ Model The graphs show the decomposition of the local expected jump risk premia in the SVCJ model. Details about the decomposition can be found in 4.2. The parameters are taken from the option-based specification where the premium on jump variance risk and/or volatility risk is restricted to be equal to zero, as described in Section 4.2 . 


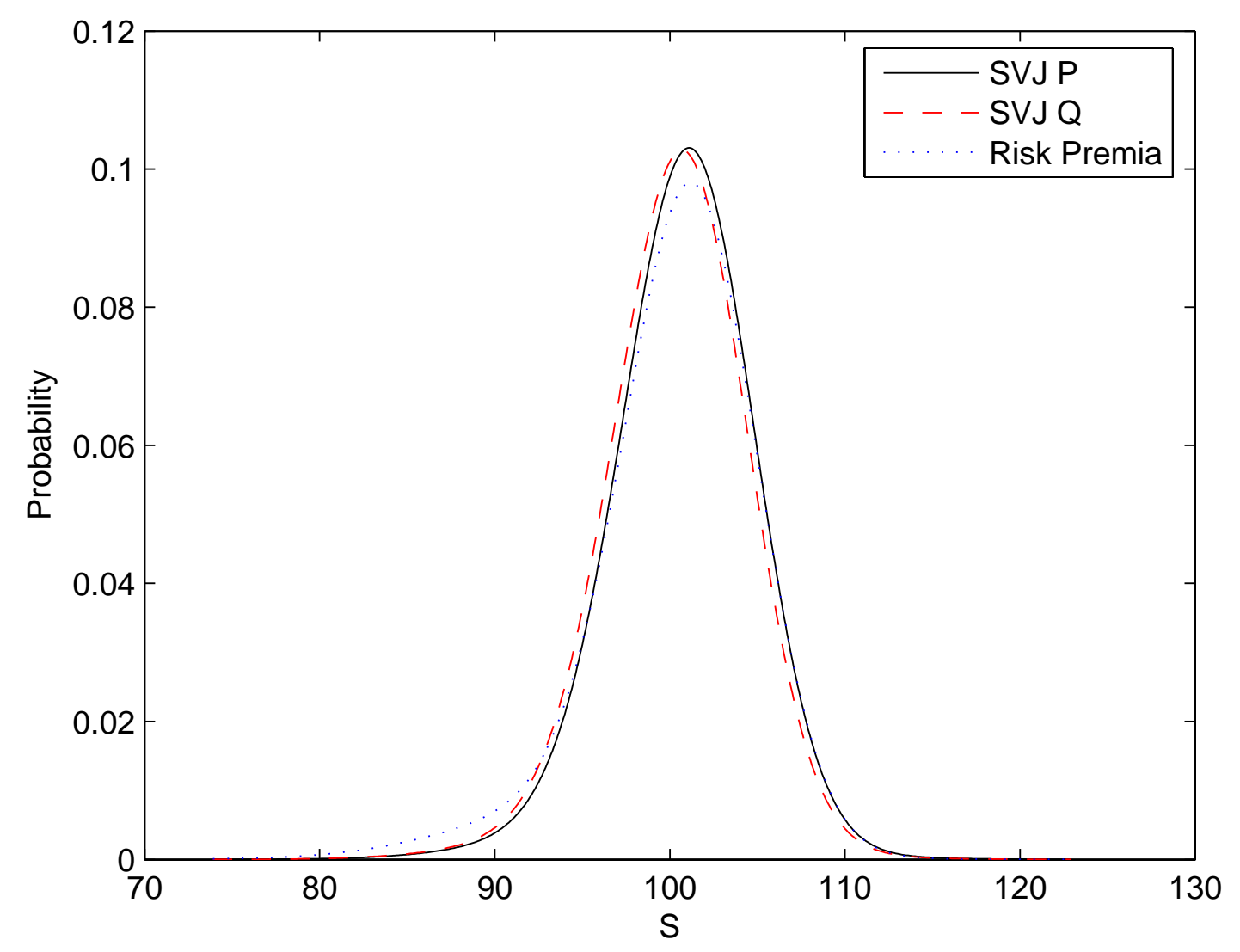

Figure 10: Densities in the SVJ Modell with and without jump risk premia The graph shows the density of the stock in the SVJ Modell under the time-series based specification. We show the density under the $\mathbb{P}$-measure as well as under the $\mathbb{Q}$-measure with and without jump risk premia. 


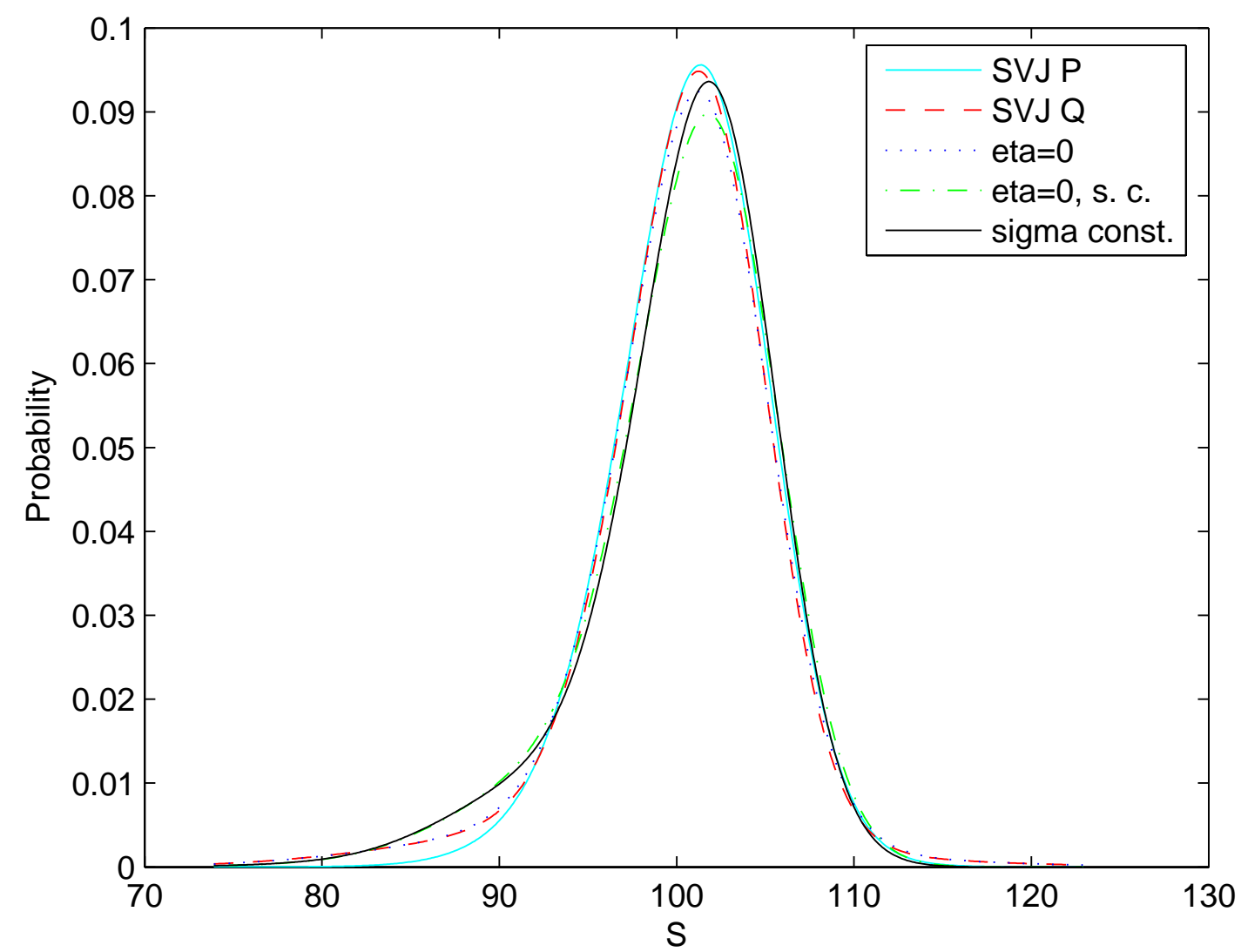

Figure 11: Densities in the SVJ Modell with and without restrictions The graph shows the density of the stock in the SVJ Modell under the optionbased specification. We show the density under the $\mathbb{P}$-measure as well as under the $\mathbb{Q}$-measure with and without restrictions on risk premia. 